University of Nebraska - Lincoln

DigitalCommons@University of Nebraska - Lincoln

\title{
$5-2017$
}

\section{The Economics of the Right to Be Forgotten}

Byung-Cheol Kim

Jin Yeub Kim

Follow this and additional works at: https://digitalcommons.unl.edu/econfacpub

Part of the Databases and Information Systems Commons, Information Security Commons, Library and Information Science Commons, and the Other Economics Commons

This Article is brought to you for free and open access by the Economics Department at DigitalCommons@University of Nebraska - Lincoln. It has been accepted for inclusion in Economics Department Faculty Publications by an authorized administrator of DigitalCommons@University of Nebraska - Lincoln. 


\title{
The Economics of the Right to Be Forgotten
}

\author{
Byung-Cheol Kim University of Alabama \\ Jin Yeub Kim University of Nebraska-Lincoln
}

\begin{abstract}
Scholars and practitioners debate whether to expand the scope of the right to be forgotten - the right to have certain links removed from search results-to encompass global search results. The debate centers on the assumption that the expansion will increase the incidence of link removal, which reinforces privacy while hampering free speech. We develop a game-theoretic model to show that the expansion of the right to be forgotten can reduce the incidence of link removal. We also show that the expansion does not necessarily enhance the welfare of individuals who request removal and that it can either improve or reduce societal welfare. Our analysis has implications for understanding the impact of the global expansion of the right to be forgotten on privacy and free speech.
\end{abstract}

\section{Introduction}

The Internet has made individuals' personal information accessible by unknown third parties with a simple Web search. Many people have concerns for their social image and reputation and want to remove their disreputable past from Internet records. Such desires raised the need for the so-called right to be forgotten (RTBF). ${ }^{1}$ The RTBF was legally established in Europe when the European Court

We gratefully acknowledge the financial support from the Networks, Electronic Commerce, and Telecommunications (NET) Institute. We appreciate the very helpful suggestions from Richard Holden and an anonymous referee. We also thank many others for helpful comments and discussions, in particular, Daniel Chen, Jay Pil Choi, Jon Eguia, Thomas Jeitschko, Doh-Shin Jeon, Michihiro Kandori, David Laband, Gong Lee, Qihong Liu, Arijit Mukherjee, Matthew Oliver, Martin Peitz, Jacopo Perego, Eric Posner, Lars Stole, and the seminar participants at Michigan State University; the Georgia Institute of Technology; Sungkyunkwan University; the University of Memphis; the University of Tokyo; Yonsei University; the 2015 International Industrial Organization Conference; the 2015 Western Economic Association International Conference; the 2015 International Conference on Game Theory; the 2015 NET Institute Conference; the 2016 Institute of Industrial Economics-Toulouse School of Economics-Institute for Advanced Study in Toulouse Conference on the Economics of Intellectual Property, Software, and the Internet; the 2016 Mannheim Center for Competition and Innovation annual conference; and the 2016 annual meeting of the Southern Economic Association.

${ }^{1}$ The right to be forgotten (RTBF) in Europe finds its intellectual roots in the French droit à l'oubli, which gives a convicted criminal the right to oppose the publication of his or her criminal history after serving time (see Rosen 2012). 
of Justice ruled in Google Spain SL, Google Inc. v. Agencia Española de Protección de Datos, Mario Costeja González (Case C-131/12, 2014 E.C.R. 317, paras. 93 and 94) that if an individual requests, search-engine operators are obliged to remove links to Web pages that contain "inadequate, irrelevant or no longer relevant, or excessive" information about that individual from the list of search results.

After this ruling, the scope of that right has become extremely controversial. Google recently expanded its compliance with the ruling to remove links from all domains in addition to its initial practice of removing them from European subdomains but only for searchers located in the country where the removal request originated (Fleischer 2016). Data regulators for the European Union are not satisfied with the limited extent of this expansion and demand that the scope of the RTBF be widened to include any domain regardless of searchers' geolocations. Thus, the extent to which the RTBF should be expanded remains in dispute.

Despite the controversy, most individuals, scholars, and policy makers approach the debate on the expansion of the RTBF similarly, as a balancing act between the allegedly competing values of privacy and free speech. The widely accepted assumption underlying the ongoing debate and recently implemented policies is that the expansion will increase the incidence of link removal and reduce the amount of information about a person that is available to others. The reduction in the availability of information reinforces privacy but weakens the free-speech interest in open access to information. However, neither scholars nor practitioners have addressed the validity of this assumption by seriously scrutinizing whether the expansion of the scope of link removals translates into more privacy and less free speech.

In this paper, we develop a game-theoretic model of interactions between a petitioner and a search engine to analyze how the probability of link removal varies when the scope of the RTBF expands to include all global domains of the search engine. In the model, the petitioner can request link removal, after which the search engine can remove the link (accept) or decline to remove the link (reject), and when the request is rejected, the petitioner can appeal to a higher authority. We use this framework to assess the critical assumptions underlying the rationales for and against global expansion of the RTBF and derive implications that are missing from the current debate.

Our analysis shows that global expansion may not necessarily induce more link removals at the margin. The key mechanism is that the expansion can change the relevant parties' payoffs that stem from link removals on a global scale. These changes may make the search engine less likely to accept the request for removal, the effect of which is a lower probability of link removal. Judging by the incidence of link removals, we see that the expansion faces an inevitable trade-off between privacy and free speech, but it could be the case that privacy is reduced and free speech is increased. The analysis also shows that the welfare of individuals who request link removals and those who seek information through links can either increase or decrease, and not necessarily in opposite directions. Hence, when accounting for the welfare of the two groups, we show that privacy and free speech need not be in conflict. Further, the expansion can either improve or reduce societal welfare. 
Taken together, these results imply that the global expansion of the RTBF should not simply be taken as a threat to free speech and access to information, nor can it be justified as an effort to increase privacy protections under the assumption that the expansion will unambiguously increase the incidence of link removals. The expansion could support the protection of data subjects' privacy without hampering the freedom of speech or the societal benefits of data sharing. This paper offers a theoretical framework to handle a nuanced consideration of the tension between privacy and free speech and thus pushes for a reformulation of the debate on the global expansion of the RTBF.

This paper relates to the enormous body of theoretical and empirical research on the economics of privacy. Acquisti, Taylor, and Wagman (2016) provide an extensive survey on this large body of literature and underline that there is no unified theory of privacy. This is because privacy issues arise in diverse contexts just as privacy itself is defined on many different dimensions such as secrecy, solitude, autonomy, and freedom. Defining privacy as secrecy, Hermalin and Katz (2006) point out two reasons why an individual might want to conceal personal information from others. First, a potential trading partner might take advantage of the information, which could lead to adverse market consequences. Second, an individual may have a taste for privacy for its own sake. The Chicago School scholars, in works such as Posner $(1978,1981)$ and Stigler $(1980)$, argue that privacy is inefficient unless individuals have a demand for privacy as a good in itself. Rather than a rebuttal to the Chicago School view, our paper incorporates this reason for privacy and expands the literature on privacy. Our analysis questions the rationale for extending the scope of the RTBF to increase privacy protections.

Both the theoretical and empirical analyses of privacy have addressed several issues on a wide variety of topics, including online advertising, price discrimination, discrimination in hiring, credit markets, and information security, among many others. ${ }^{2}$ The existing literature on privacy is mostly concerned with the market and welfare effects of protecting and disclosing personal information. While our research also speaks to the informational consequences and efficiency effects of expanding the RTBF, the primary focus is to clearly identify the fundamental forces at work in the mechanics of link removals and thus to assess the validity of the presumption in the debate on the expansion of the RTBF.

The remainder of the paper is organized as follows: Section 2 provides background on the landmark decision of the European court and the process of requesting link removals. Section 3 presents a model that captures this situation and characterizes equilibria. Section 4 provides the equilibrium properties of global expansion, focusing on its impact on the equilibrium probability of link removal. Section 5 studies the welfare properties of a global expansion, discussing its impact on social welfare. Section 6 offers concluding comments. The appendix contains proofs for equilibrium characterization in the game and for comparative

\footnotetext{
${ }^{2}$ For example, Kovbasyuk and Spagnolo (2016) study the effects of erasing past records of feedback in a dynamic market with heterogeneous sellers of unknown quality and buyers, which has implications for the RTBF.
} 
statics results. The online appendix provides additional details and discussions that supplement our results. ${ }^{3}$

\section{Background}

In 2009 Mario Costeja González, a Spanish lawyer, requested that Google Spain remove a link to a digitized 1998 article in the newspaper La Vanguardia about the forced sale of his property arising from social security debts. His grounds for requesting the removal of the link were that the forced sale had been concluded years before, that the debt had been paid in full, and that information regarding his home foreclosure was no longer relevant but defamatory. When the request was unsuccessful, Costeja sued Google Inc. The case was eventually elevated to the European Court of Justice. In May 2014, the court found for Costeja and ordered both Google Inc. and its subsidiary Google Spain to erase the pertinent links from Google's search results for Costeja's name (Case C-131/12, 2014 E.C.R. 317).

After the European RTBF ruling, Google launched an online request process. ${ }^{4}$ Any individual in the European Union and the European Free Trade Association (EFTA) member countries can make a request for the removal of links by filling out a Web form provided on Google's website. For each submitted request, Google evaluates whether the search results include outdated or inaccurate information about the person and weighs whether the general public has a preponderant interest in having access to the information in question. If Google declines to remove certain links, an individual can request a local data-protection authority to review Google's decision. Such data-protection authorities include the Information Commissioner's Office (ICO) in the United Kingdom, the Agencia Española de Protección de Datos (AEPD) in Spain, and the Commission Nationale de l'Informatique et des Libertés (CNIL) in France. If the data-protection authority finds for the requester after reviewing the issues relevant to the links in question, Google is informed that it must remove the links.

Since the launch of the request process, Google has received more than 710,000 link removal requests from individuals in the European Union and the EFTA. Of the almost 2 million URLs that have been evaluated, 43.1 percent have been removed, while the rest of the requests were rejected. ${ }^{5}$ Many individuals objected

\footnotetext{
${ }^{3}$ In the online appendix, we show that our results for the impact of changing the scope of the RTBF remain qualitatively intact in the complete-information game and provide illustrations and detailed discussions of our main argument and the comparative statics analysis with respect to appeal costs.

${ }^{4}$ Yahoo Inc. and Microsoft also started to take requests for search-result removals from Yahoo Search and Bing. As Google's search market share in Europe dominates Bing, Yahoo, and others by more than 90 percent according to StatCounter, Search Engine Market Share in Europe (http:// gs.statcounter.com/search-engine-market-share/all/europe), we focus on Google as a representative search provider with regard to the issue of the RTBF in Europe.

${ }^{5}$ The statistics reported here are accurate as of April 22, 2017. For up-to-date data, see Google, Transparency Report (https://transparencyreport.google.com/eu-privacy/). Google does not share specific statistics on the types of requests it receives from individuals, but evidence exists that the majority of requests are from citizens who are trying to protect their personal and private information rather than from criminals, politicians, and public figures (see, for example, Tippmann and Powles 2015).
} 
to Google's decisions and subsequently filed appeals to local data-protection authorities for review. For example, in the year after the ruling, the ICO handled over 180 complaints from individuals who disagreed with Google's rejection of their request. The ICO contended that Google had correctly rejected about three-quarters of them but did not agree with Google's assessment in 48 cases and asked Google to revise its decision (Rawlinson 2015).

At first, Google restricted its compliance with the RTBF ruling by removing links from search results only in its European domains; links remained in non-European domains, including Google.com. In September 2014, EU data regulators published guidelines calling on Google to apply the ruling to all of its global domains. They argued that local delisting was not effective and complete protection of data subjects' rights to erasure of data. Google nevertheless refused to widen the scope of its delisting, arguing that the European RTBF ruling is not binding beyond the European Union's jurisdiction. In early 2016, Google expanded its compliance to also remove links from all domains including Google.com but only for searches conducted in the country of the person requesting the removal. Expansion such as this still does not go as far as the data regulators have demanded.

\section{The Model}

\subsection{Setup}

We consider a game in which two parties are in a potential conflict over the removal of links, referred to as an RTBF game. A petitioner (P) suffers harm of $h>0$ from the presence of links to information about him provided on all domains of a Web search engine $(\mathrm{G}){ }^{6}{ }^{6}$ The parameter $h$ can include either pecuniary or nonpecuniary damage to P's reputation or any subjective disutility from the fact that defamatory links are publicly available in an online search. When links are removed only from a subset of domains, $\mathrm{P}$ is not perfectly compensated for his harm but recovers a positive fraction $q$ of his harm. The parameter $q$ can be interpreted as measuring how the petitioner values the limited deletion, so $q h$ represents the amount of salvageable harm from restricted link removal. ${ }^{7}$ Search engine $\mathrm{G}$ incurs a net loss of size $l>0$ from removing the links relative to keeping them, which may vary depending on the scope of the removal. ${ }^{8}$ The parameter $l$ captures the costs that arise from removing the links, such as administrative costs, or a shrinkage of the user base due to the removed links.

Petitioner P can claim the removal of links at a nonmonetary cost of $c>0$. If $G$ accepts the claim, then she loses $l$, and $\mathrm{P}$ receives $-(1-q) h-c$. If G rejects the claim, then $\mathrm{P}$ must decide whether to give up or appeal (that is, file a complaint)

\footnotetext{
${ }^{6}$ For clarity of exposition, we use male pronouns for P and female pronouns for G. Search engine $\mathrm{G}$ can represent any data operator, including Google, that is subject to the RTBF ruling.

${ }^{7}$ The parameters $q$ and $h$ can vary across the petitioners. The size of these parameters may depend on the search intensity, the nature of the searched information, or individual characteristics of the petitioner (for example, whether he is a public figure, his privacy sensitivities and attitudes, and so on).

${ }^{8}$ We allow for unrestricted correlation structures across the parameters $q, h$, and $l$.
} 


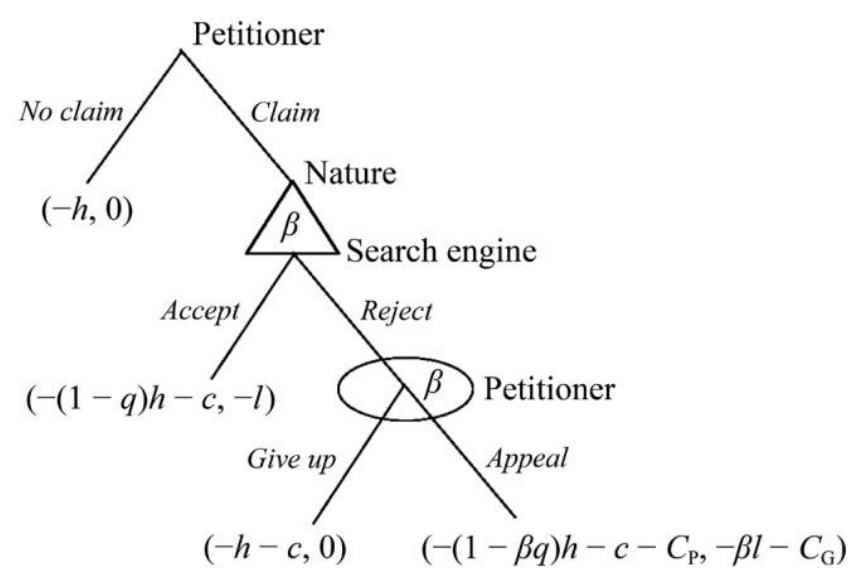

Figure 1. The game tree

to a local data-protection authority. Petitioner P's appeal involves costs for both parties, summarized by $C_{\mathrm{P}}>0$ for $\mathrm{P}$ and $C_{\mathrm{G}}>0$ for $\mathrm{G}$. With probability $\beta, \mathrm{P}$ wins on appeal, and the data-protection authority orders $\mathrm{G}$ to remove the links. The removal order is assumed to be fully enforceable, and no further action can be taken by either $\mathrm{P}$ or $\mathrm{G}$ following a ruling. ${ }^{9}$ Then the expected payoffs from the appeal are $-(1-\beta q) h-c-C_{\mathrm{P}}$ for $\mathrm{P}$ and $-\beta l-C_{\mathrm{G}}$ for $\mathrm{G}$.

In the shadow of a ruling by an external authority, the players will form some assessments of the expected ruling characterized by $\beta$. We assume that $\mathrm{G}$ can make a better assessment of the expected ruling while $P$ may not have the expertise or experience to do so. In particular, $G$ estimates the likelihood of $P$ prevailing on appeal to be $\beta$; we refer to $\mathrm{G}$ with estimate $\beta$ as being of type $\beta$. Petitioner $\mathrm{P}$ believes that $\beta$ is drawn from a nondegenerate distribution $F(\cdot)$ over the interval $[0,1]$ with density $f(\cdot)$. In our RTBF game, all of the parameters except $\beta$ are common and public knowledge. The game tree in Figure 1 depicts the sequence of events with the expected payoffs specified, where P decides whether to claim and whether to give up or appeal without knowing $\beta .^{10}$

Before proceeding to characterize the equilibrium, we briefly provide the basis for our assumption of incomplete information. There is a practical reason why incomplete information is necessary for representing the current situation. Under

\footnotetext{
${ }^{9}$ In reality, the search engine can still refuse to remove the links despite further enforcement action or fines it might face should it not accept the data-protection authority's decision (see, for example, Thom 2015). Both the petitioner and the search engine can also appeal the decision of the data-protection authority to a higher authority. We assume away those possibilities for simplicity and tractability of analysis without losing the key dimensions of the dispute. See Section 4.3 for an elaboration of this point.

${ }^{10}$ The sequence of our game and the subsequent characterization of equilibrium resemble those à la Bebchuk (1984) and Nalebuff (1987). The important difference is that they focus on pretrial settlement negotiation. We rule out the possibility of a settlement demand by a petitioner because pretrial bargaining opportunities are not prevalent in the cases of the RTBF.
} 
complete information, in any equilibrium that involves $\mathrm{P}$ claiming, one of two cases arises depending on the parameter values: in the first case, G always accepts the claim in anticipation of P's equilibrium strategy of appealing; in the second case, $\mathrm{G}$ always rejects, and $\mathrm{P}$ always appeals on the equilibrium path. But under complete information, there is no equilibrium that can represent the real cases in which individuals do not appeal to local data-protection authorities when their claims are rejected by the search engine (see Section 2). Further, some individuals with weak cases may claim nonetheless in the hopes of winning on appeal if rejected, precisely because of the uncertainty over the expected decision. Beyond the practicality of the model, the presence of asymmetric information does not drive the results in this paper. More important, we incorporate one-sided incomplete information for the key reason that the search engine can be thought of as a repeat player in the sense that it has encountered the precedents of many other petitioners' cases. ${ }^{11}$ Although our game is not modeled as a repeated game, nor does the start of our game represent any specific point in time since the RTBF ruling, it seems more plausible to assume that the search engine has a superior assessment of the appeal's expected outcome. ${ }^{12}$

\subsection{Equilibrium}

We first consider the subgame following the petitioner's claim. We represent P's strategy in the subgame by $p_{\mathrm{a}}$, which indicates his probability of appealing if the claim is rejected. When $\mathrm{G}$ has anticipated that $\mathrm{P}$ will choose to appeal according to $p_{\mathrm{a}}$, a $\mathrm{G}$ of type $\beta$ compares the payoff from accepting $-l$ with the expected payoff from rejecting $\left(1-p_{\mathrm{a}}\right) \times 0+p_{\mathrm{a}}\left(-\beta l-C_{\mathrm{G}}\right)$. Because the difference between G's payoff from accepting and the expected payoff from rejecting is strictly increasing in $\beta$, no matter what P's action is, G's higher types find acceptance relatively more attractive than lower types do. Thus, G's best response against any strategy of $\mathrm{P}$ in the subgame is to accept the claim if $\beta>\beta_{\mathrm{G}}$ and to reject the claim if $\beta \leq \beta_{\mathrm{G}}$, where $\beta_{\mathrm{G}}$ is the cutoff type of $\mathrm{G}$ that is indifferent to accepting or rejecting the claim given $p_{\mathrm{a}}$; that is, $\beta_{\mathrm{G}}=\left(l / p_{\mathrm{a}}-C_{\mathrm{G}}\right) / l .^{13}$

Petitioner $\mathrm{P}$ at his decision node after the claim has been rejected forms a posterior expectation of $\beta$ with his posterior beliefs concentrated on $\left[0, \beta_{\mathrm{G}}\right]$, given by $\mathbb{E}\left[\beta \mid \beta \leq \beta_{\mathrm{G}}\right]=\int_{0}^{\beta_{\mathrm{G}}}\left[x f(x) / F\left(\beta_{\mathrm{G}}\right)\right] d x$. Accordingly, $\mathrm{P}$ compares his payoff from giving up, $-h-c$, with the expected payoff from appeal, $-(1-\mathbb{E}[\beta \mid \beta \leq$ $\left.\left.\beta_{\mathrm{G}}\right] q\right) h-c-C_{\mathrm{P}}$. This expected payoff increases with $\beta_{\mathrm{G}}$ because the posterior expectation $\mathbb{E}\left[\beta \mid \beta \leq \beta_{\mathrm{G}}\right]$ is a monotonically increasing function of $\beta_{\mathrm{G}}$. We define $\beta^{*}$ to be a unique value of $\beta_{\mathrm{G}}$ that solves $\mathbb{E}\left[\beta \mid \beta \leq \beta^{*}\right] q h=C_{\mathrm{P}}$; that is, $\beta^{*}$ is the

\footnotetext{
${ }^{11}$ We thank an anonymous referee for making this point.

${ }^{12}$ One might then think of the case in which incomplete information is two sided; for example, both players have uncertainty about the likelihood of prevailing, or each player has uncertainty about the magnitude of the other player's harm or loss. Our results are not derived from the particular information structure, and we do not lose any important economic reasoning by assuming away the possibility of the petitioner's private information.

${ }^{13}$ We assume that the indifferent type rejects.
} 
cutoff value such that rejection by the types below $\beta^{*}$ makes $\mathrm{P}$ indifferent between giving up and appealing. Then P's best response at rejection by Gs of type $\beta$ $\leq \beta_{\mathrm{G}}$ is $p_{\mathrm{a}}=1$ if $\beta_{\mathrm{G}}>\beta^{*}, p_{\mathrm{a}} \in[0,1]$ if $\beta_{\mathrm{G}}=\beta^{*}$, and $p_{\mathrm{a}}=0$ if $\beta_{\mathrm{G}}<\beta^{*}$.

We characterize two conditions that rule out the cases in which $\mathrm{P}$ always gives up at rejection regardless of his posterior expectations and in which $G$ always accepts the claim no matter what her type is if she believes that $\mathrm{P}$ will appeal with a probability of 1 .

Condition 1. This condition specifies that $\mathbb{E}[\beta] q h \geq C_{\mathrm{P}}$.

Condition 1 implies that $\mathrm{P}$ prefers appealing over giving up even if all types of $\mathrm{G}$ reject. In that case P's posterior belief is identical to his prior belief regarding G types. ${ }^{14}$

Condition 2. This condition specifies that $l>C_{\mathrm{G}}$.

Condition 2 necessarily guarantees that some types of $\mathrm{G}$ will always reject, that is, $F\left(\beta_{\mathrm{G}}\right)>0$, for any given $p_{\mathrm{a}}>0$. Let $\beta_{\mathrm{G}}^{*}$ be the cutoff type of $\mathrm{G}$ that is indifferent between accepting or rejecting the claim when $\mathrm{G}$ believes that $\mathrm{P}$ will appeal with certainty at rejection. That is, $\beta_{\mathrm{G}}^{\star} \equiv\left(l-C_{\mathrm{G}}\right) / l$.

Proposition 1. Under conditions 1 and 2, there is a unique Nash equilibrium in the subgame after P's claim, in which Gs of type $\beta>\beta_{\mathrm{G}}$ accept the claim while Gs of type $\beta \leq \beta_{\mathrm{G}}$ reject it and $\mathrm{P}$ appeals with probability $p_{\mathrm{a}}>0$, where $\beta_{\mathrm{G}}$ and $p_{\mathrm{a}}$ satisfy the following conditions:

i) if $\beta_{\mathrm{G}}^{\star}>\beta^{\star}$, then $\beta_{\mathrm{G}}=\beta_{\mathrm{G}}^{\star}$ and $p_{\mathrm{a}}=1$;

ii) if $\beta_{\mathrm{G}}^{\star} \leq \beta^{*}$, then $\beta_{\mathrm{G}}=\beta^{*}$ and $p_{\mathrm{a}}=l /\left(\beta^{*} l+C_{\mathrm{G}}\right) \in(0,1]$.

We explain the intuition for proposition 1 as follows. First, for the case of $\beta_{\mathrm{G}}^{\star}>\beta^{\star}$, at rejection by Gs of type $\beta \leq \beta_{\mathrm{G}}^{\star}$, filing an appeal is profitable to $\mathrm{P}$ because $\mathbb{E}\left[\beta \mid \beta \leq \beta_{\mathrm{G}}^{*}\right] q h>\mathbb{E}\left[\beta \mid \beta \leq \beta^{\star}\right] q h=C_{\mathrm{P}}$; hence, P's optimal strategy $p_{\mathrm{a}}=1$ and G's optimal cutoff type $\beta_{\mathrm{G}}^{\star}$ are justified. By contrast, if $\beta_{\mathrm{G}}^{\star} \leq \beta^{\star}$, then P's commitment to appeal is not credible because it induces rejection by Gs of type $\beta \leq \beta_{\mathrm{G}}^{*}$, which in turn makes P's appeal unprofitable because $\mathbb{E}\left[\beta \mid \beta \leq \beta_{\mathrm{G}}^{\star}\right] q h \leq \mathbb{E}\left[\beta \mid \beta \leq \beta^{\star}\right] q h=C_{\mathrm{P}}$. Hence, $\mathrm{P}$ lowers his probability of appealing so as to induce Gs of type $\beta \in\left(\beta_{\mathrm{G}}^{*}, \beta^{\star}\right]$ to also reject. Petitioner P now faces a greater chance of being rejected, but after rejection by Gs of type $\beta \leq \beta^{*}$, $\mathrm{P}$ is indifferent between appealing and giving up, given the higher posterior expectation of prevailing, which confirms his optimal strategy $p_{\mathrm{a}} \in(0,1]$. The case $\beta_{\mathrm{G}}^{*}=\beta^{*}$ is a special one in which $\beta_{\mathrm{G}}=\beta^{*}$ and $p_{\mathrm{a}}=1$.

We now consider P's initial node in which he has to decide whether to claim. Given the subgame equilibrium strategies, P's optimal strategy at the initial node is to claim if his expected payoff from claiming is greater than his payoff from not claiming. This condition reduces to

$$
c \leq\left[1-F\left(\beta_{\mathrm{G}}\right)\right] q h+F\left(\beta_{\mathrm{G}}\right) p_{\mathrm{a}}\left[\mathbb{E}\left[\beta \mid \beta \leq \beta_{\mathrm{G}}\right] q h-C_{\mathrm{P}}\right],
$$

${ }^{14}$ Condition 1 is equivalent to the assumption in Nalebuff (1987) that P's case has merit. 
where we assume that $\mathrm{P}$ chooses to claim when expression (1) holds as equality. The reasoning is straightforward: the claim's cost has to be small enough for claiming to be profitable to $\mathrm{P}$, assuming that all of the moves after the claim are determined according to the strategies specified in proposition 1 . We can summarize the analysis as follows.

Proposition 2. Under conditions 1 and 2, there is a unique sequential equilibrium in which P claims if and only if expression (1) holds; P and G use the subgame strategies described in proposition 1.

Proposition 2 implies that as long as the claim's cost is small enough, a petitioner with sufficient harm will request the removal of defamatory links. The petitioner does so in the hope that the search engine will accept his request, and if the search engine rejects the request, the petitioner expects to win on appeal with a positive probability. Both of these scenarios lead to the removal of the links.

In the case in which condition 1 fails, there is still a unique sequential equilibrium in which $\mathrm{P}$ never claims, all Gs reject, and $\mathrm{P}$ always gives up at rejection. If condition 1 holds but condition 2 fails, there are two equilibria to the subgame following P's claim: one subgame equilibrium in which some Gs reject and $\mathrm{P}$ appeals with positive probability (as in proposition 1.ii) and a second subgame equilibrium in which all Gs accept and P always appeals. ${ }^{15}$ Hence, condition 1 gives a necessary condition for the possibility that an appellate review by a dataprotection authority arises as an equilibrium outcome; condition 2 guarantees a unique sequential equilibrium in the RTBF game.

The equilibrium characterization under these two conditions gives a reasonable approximation of Europe's current situation with regard to the RTBF. After the European ruling, Google received a considerable number of removal requests, some of which were rejected and whose petitioners then appealed (see Section 2). Further, when we note that the request process is easily accessible-individuals need only complete an online form on Google's website and do not pay a monetary fee-the claim's cost $c$ in our model can be assumed to be very small.

\section{Equilibrium Properties of the Global Expansion}

In this section, we examine the effect of a change in the parameters $q$ and $l$ on the probability that the links are removed in equilibrium. These comparative statics results have implications for the debate over the global expansion of the RTBF, which we discuss.

\subsection{Comparative Statics of the Equilibrium Probability of Link Removal}

The removal of links as a resulting equilibrium outcome occurs only if a claim is made. Hence, we focus on the sequential equilibrium in which $\mathrm{P}$ claims for given parameter values, which we refer to as a claim equilibrium. In the claim

\footnotetext{
${ }^{15}$ See Section A4 for formal characterizations of equilibria in the absence of condition 1 or 2.
} 
equilibrium, the links are removed either when $G$ accepts the claim or when $G$ rejects but $\mathrm{P}$ appeals and wins. In equilibrium, the total prior probability that $\mathrm{G}$ accepts the claim is $1-F\left(\beta_{\mathrm{G}}\right)$, and the probability that $\mathrm{P}$ appeals is $p_{\mathrm{a}}$, with $\mathrm{P}$ 's expected probability of winning at $\mathbb{E}\left[\beta \mid \beta \leq \beta_{\mathrm{G}}\right]$. Thus, the equilibrium probability of link removal is given by

$$
\operatorname{Pr}(\text { Link Removal }) \equiv\left[1-F\left(\beta_{\mathrm{G}}\right)\right]+F\left(\beta_{\mathrm{G}}\right) p_{\mathrm{a}} \mathbb{E}\left[\beta \mid \beta \leq \beta_{\mathrm{G}}\right] .
$$

To formalize the comparative statics results, let $q^{*}$ and $l^{*}$ denote the values of $q$ and $l$, respectively, such that $\beta_{\mathrm{G}}^{*}=\beta^{*}$, and let $q$ denote the lower bound of $q$ implied by condition 1 , each defined given the other primitives. The following proposition describes the effects of changing the proportion of P's harm recovered by removal $(q)$ or the amount of G's loss at stake $(l)$ on the equilibrium probability of link removal in the claim equilibrium under conditions 1 and 2.

\section{Proposition 3.}

i) The equilibrium probability of link removal is increasing in $q$ if $q \in\left[\underline{q}, q^{*}\right)$ but is constant in $q$ if $q \geq q^{*}$.

ii) The equilibrium probability of link removal is increasing in $l$ if $l \in\left(C_{\mathrm{G}}, l^{*}\right)$ but is decreasing in $l$ if $l \geq l^{*}$.

Proposition 3 is shown by the solid lines in Figures 2 and $3 .{ }^{16}$ We give the reasoning behind proposition 3 for each case as follows.

Figure 2 shows the effect of a change in the proportion of P's harm recovered by removal $(q)$. When $q \in\left[q, q^{*}\right), \mathrm{P}$ appeals with some positive probability $p_{\mathrm{a}}$ $<1$ on rejection by Gs of type $\beta \leq \beta^{\star}$. If $\mathrm{P}$ can save more by removal (that is, a higher $q$ ), then his desire to appeal becomes greater, so he appeals more often (higher $p_{\mathrm{a}}$ ), which induces more Gs to accept (higher $1-F\left(\beta^{\star}\right)$ ). At the same time, $\mathrm{P}$ expects that he is less likely to win an appeal (lower $\mathbb{E}\left[\beta \mid \beta \leq \beta^{*}\right]$ ), so the probability that $\mathrm{P}$ appeals and wins in equilibrium might increase or decrease. Regardless, P's winning on appeal occurs, in expectation, on a subset of reduced rejection possibility. Thus, the effect of a higher probability of G's acceptance contributes to a greater chance of the links being removed. On the other hand, when $q \geq q^{*}$, P appeals with a probability of 1 , so a higher $q$ makes proceeding to an appeal even more profitable. So G's optimal cutoff type remains the same, which leaves the probability of G's acceptance or rejection, as well as P's posterior expected probability of winning, unchanged. Hence, any change in $q$ does not affect the equilibrium probability of link removal.

Figure 3 shows the effect of a change in the amount of G's loss (l); the dotted lines indicate the probabilities of link removal in two possible claim equilibria when condition 2 fails. When $l \in\left(C_{\mathrm{G}}, l^{*}\right)$, Gs of type $\beta \leq \beta^{*}$ reject, which in turn makes $\mathrm{P}$ indifferent between giving up and appealing. A higher $l$ increases $\mathrm{G}^{\prime} \mathrm{s}$

${ }^{16}$ Figures 2 and 3 are obtained for a simple example that assumes a uniform distribution $F(\beta)$ on $[0,1], h=50, C_{\mathrm{P}}=C_{\mathrm{G}}=10$, and $l=50$ for Figure 2 and $q=.9$ for Figure 3 . The graphs are generic as long as $c$ satisfies expression (1) given the subgame equilibrium strategies for any other parameters that satisfy conditions 1 and 2 . 


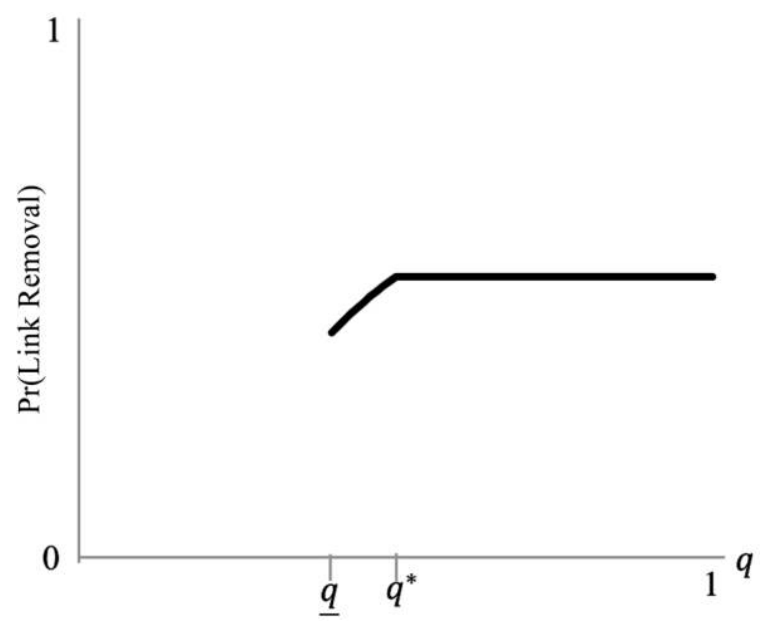

Figure 2. The effect of $q$ on the probability of link removal in the claim equilibrium under conditions 1 and 2 .

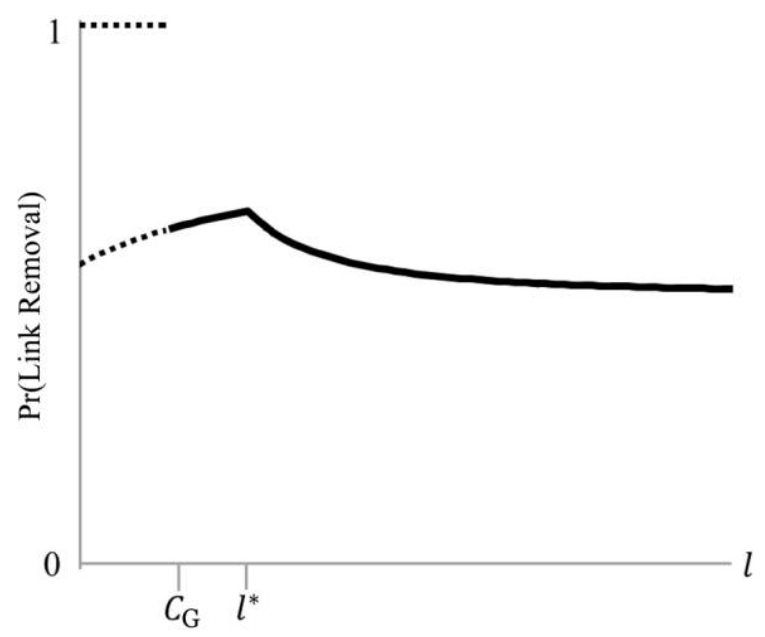

Figure 3. The effect of $l$ on the probability of link removal in the claim equilibrium under conditions 1 and 2 .

expected net gain from rejecting relative to accepting, but $\mathrm{P}$ adjusts his probability of appealing upward so as to maintain rejection by Gs of types below $\beta^{\star}$. Hence, the probability of G's rejection (or acceptance) and P's posterior expected probability of prevailing remain unchanged, whereas the probability that $\mathrm{P}$ appeals on rejection increases. This increase in $p_{\mathrm{a}}$ leads to an increase in the equilibrium probability of link removal. On the other hand, when G's loss from link removal is high enough that $l \geq l^{*}$, rejection by Gs of type $\beta \leq \beta_{\mathrm{G}}^{*}$ induces $\mathrm{P}$ to 


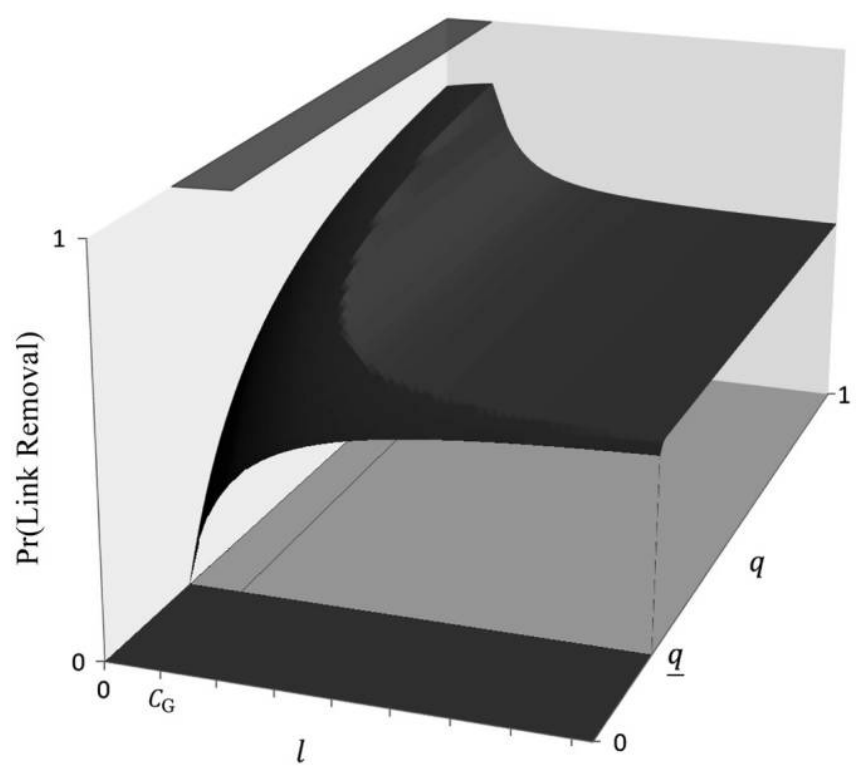

Figure 4. The effects of $q$ and $l$ on the equilibrium probability of link removal

appeal with a probability of 1 in the expectation that his chance of prevailing is high. If $\mathrm{G}$ loses more by removal (that is, a higher $l$ ), then more Gs reject (lower $\left.1-F\left(\beta_{\mathrm{G}}^{*}\right)\right)$. Given a higher probability of rejection, $\mathrm{P}$ expects a greater chance of prevailing (higher $\mathbb{E}\left[\beta \mid \beta \leq \beta_{\mathrm{G}}^{*}\right]$ ); he still maintains his commitment to appeal $\left(p_{\mathrm{a}}=1\right)$. However, link removal occurs with a probability of less than 1 on G's rejection, while it always ensues on G's acceptance. The chance of link removal is reduced with G's less likely acceptance by more than it is increased with P's higher likelihood of winning on appeal, which leads to a decrease in the equilibrium probability of link removal.

Figure 4 shows how changes in both $q$ and $l$ affect the equilibrium probability of link removal. ${ }^{17}$ For the region where $q \geq q$ and $l>C_{\mathrm{G}}$, the surface shows the probability of link removal in the unique equilibrium in which $\mathrm{P}$ claims. The pair $(q, l)$ at the kink on that surface satisfies $\beta_{\mathrm{G}}^{\star}=\beta^{\star}$ given other parameter values. For the region where $q \geq q$ and $l \leq C_{\mathrm{G}}$, the two surfaces are drawn to reflect the probabilities of link removal in two possible claim equilibria. The dark area on the $(q, l)$-plane indicates that $\mathrm{P}$ never claims in the unique sequential equilibrium when $q<q$, and so the probability of link removal is 0 .

\subsection{Effects of the Global Expansion on Link Removals}

Privacy watchdogs in the European Union have called on Google to apply the European ruling to its global search results, not only to local subdomains. Al-

${ }^{17}$ Figure 4 is obtained in an example with a uniform $F(\cdot), h=50$, and $C_{\mathrm{P}}=C_{\mathrm{G}}=5$. 
though Google extended its compliance in early 2016, link removal is still limited to the domains that are accessible in the data subject's country, and only for searchers in the same country as the data subject. The debate over whether to expand the European ruling to all of Google's global domains without restrictions-what we call global expansion-is ongoing.

Underlying the debate is the supposition that expanding the scope of the RTBF to all domain extensions of the search engine will unambiguously cause more link removals at the margin. The advocates of global expansion who frame the issue in this way argue that expansion is a means to strengthen the protection of privacy as represented by a higher incidence of link removal. Those who oppose the expansion fear that it poses a threat to freedom of speech and access to information because the expansion will result in the deletion of more links that could help search-engine users easily find desired information.

However, both sides of the debate fail to account for the way that global expansion of the RTBF may change the payoffs for the petitioner and the search engine if the links are removed from all domains. When the removal of the links is applied in an unrestricted manner, it is reasonable to expect that the petitioner will save the (nearly) entire harm. Furthermore, the search engine's loss from the removal is likely to be larger. ${ }^{18}$ The following assumption formally translates the changes in the players' payoffs by the expansion into our model.

Assumption 1. The global expansion is represented by a combination of an upward shift in $q$ to 1 and an increase in $l$.

Assumption 1 approximates how global expansion will change the players' payoffs. Those changes in payoffs, in combination, will influence the behaviors of potential petitioners and search engines. If this connection is taken into account, then the expansion of the scope of link removals might not lead to the verbatim expansion of the link removals.

Figure 4 shows that for the region where $q \geq q$ and $l>C_{\mathrm{G}}$, if a proportion of harm that the petitioner can recover from limited link removal is sufficiently low (that is, $q<q^{*}$ given $l$ ), then increases in the petitioner's salvageable proportion of harm and in the search engine's loss might lead to a higher probability of link removal, depending on the magnitude of those increases. However, if the petitioner can recover a certain level of proportion of harm by limited removal (that is, $q \geq q^{*}$ given $l$ ), then a larger loss for the search engine for any increase in the petitioner's salvageable proportion contributes to a lower chance of link removal in equilibrium. This decrease is essentially due to a lower probability that the search engine accepts the claim. These discussions lead to our main argument.

Corollary 1 . Under assumption 1 , the global expansion has the following effect on any claim associated with $q \geq \underline{q}$ and $l>C_{\mathrm{G}}$ : the probability of link re-

${ }^{18}$ Removing links on all domains "will result in significant increase in technical work and related administrative costs" (Gilbert 2014). Also see Munson (2015), which points out that "[n]ot only could [delisting on all domains] open Google up to severe financial risk, it could also represent a massive increase in workload for a company." 
moval may increase for petitioners with a sufficiently low $q$, but it will decrease for those with a relatively high $q$.

Corollary 1 shows the impact of the global expansion in the intensive margin, which corresponds to the way that global expansion will change the possibility that a petitioner's claim to remove links will result in the removal, should he claim to remove links even under the limited scope of removal. ${ }^{19}$ The expansion will also generate effects in the extensive margin. For example, petitioners who had not claimed under the limited scope of removal might claim after the global expansion, which contributes to more incidence of link removal; the search engine that would have accepted a claim before the expansion might want to reject it after the expansion, which reduces the incidence of link removal. ${ }^{20}$ By assuming some appropriate distributions of all payoff-relevant parameter values across all potential removal claims, we can measure the effect of the expansion on the overall incidence of link removals. Gauging this measure complicates the exposition without producing unambiguous comparative static results. Our analysis yields equivocal results, but what is essential is that the global expansion of the RTBF can lessen the possibility of each removal claim resulting in the removal of the links. Accordingly, the expansion of the scope of the RTBF might not necessarily increase the incidence of link removals.

\subsection{Implications and Discussion}

Participants in the RTBF debate have framed global expansion as supporting data subjects' privacy while threatening freedom of speech and open access to information. This perspective is legitimate only if global expansion is desirable for privacy but undesirable for free speech, where desirability is evaluated according to some appropriate measure. If the probability of link removal is the measure, then an increased incidence of link removals represents an increase in privacy and a decrease in free speech. While it has been assumed that global expansion will render more link removals at the margin, our theoretical analysis suggests that expansion can decrease the incidence of link removals. In this sense, global expansion can unexpectedly be undesirable for privacy but desirable for free speech.

One implication of our analysis is that the expansion cannot be justified as an effort to strengthen privacy protections, nor is it necessarily a threat to the right

\footnotetext{
${ }^{19}$ We note that under the current practice of removing links by Google, individuals are not given the option of decoupling their link removal requests to remove only from certain domains so as to prevent their requests being rejected.

${ }^{20}$ A petitioner does not claim in equilibrium either when his salvageable proportion of harm $(q)$ is sufficiently low or when his claim's cost $(c)$ is sufficiently high. The first case corresponds to when condition 1 fails so that appealing is never a weakly dominant strategy for P; hence, no claim constitutes the unique equilibrium. Even when condition 1 holds, the second case arises if the condition for P's decision to claim expression (1) is not satisfied so that no claim is in the unique equilibrium. The latter case may not be prevalent because the online request process is low cost while the appeal might be costly. Finally, the case of $\mathrm{G}$ always accepting regardless of her type can arise when condition 2 fails (that is, $l \leq C_{\mathrm{G}}$ ).
} 
of free speech and access to information. The assumption that the expansion will unambiguously increase the incidence of link removal is unfounded. Thus, the debate about global expansion should not be framed as a clash between reinforcing privacy, as represented by a higher incidence of link removal, and protecting free speech, as represented by a lower incidence of link removal.

Before turning to the analysis of welfare properties, we discuss how our model captures the key dimensions of the RTBF dispute and global expansion. We model the dispute as a game between a search engine and a single individual petitioner that ends with an expectation of a ruling by a data-protection authority (DPA) if the petitioner appeals. The simplicity of the model may raise some doubts about the relevance of our model to the details of the RTBF dispute process.

First, our model does not directly illustrate scenarios in which the petitioner or the search engine appeals the decision of the DPA by bringing the case before a higher authority or in which the search engine refuses to carry out the DPA's decision to remove the links, after which the petitioner may take further legal action. Consideration of these scenarios can be easily accommodated by amending the game tree to include additional sequential actions of the players. ${ }^{21}$ This extension only complicates the analysis without adding commensurate insight. Perhaps a simpler way to capture those scenarios is by enlarging the definition of the parameters $C_{\mathrm{G}}$ and $C_{\mathrm{P}}$ to reflect the levels of expenditures that the players expect to spend should further actions take place beyond the petitioner's appeal to the DPA.

Second, one might argue that when the search engine accepts a single request submitted by a petitioner, it does not impose a significant loss on the search engine (low l), so the dispute should rather be described as a game between a search engine and many potential petitioners. This argument is compelling if a case brought before a supervisory or judicial authority is publicized. In such a situation, the real losses to the search engine might occur if a petitioner's win in court attracts many more petitioners with similar removal requests that need to be accepted immediately. ${ }^{22}$ Then $C_{\mathrm{G}}$ should include the expected aggregate losses from all ensuing cases and any foreseeable indirect costs that are not captured by the loss $l$ of the initial case when an additional verdict arises. ${ }^{23}$ In a similar vein, further legal action can cost the petitioner in extra time, legal fees, or even psychic harm. For example, a petitioner's identity often gets more attention in the

${ }^{21}$ For example, in Figure 1 after $\mathrm{P}$ appeals, a chance node of $\mathrm{P}$ winning or losing can be added. If $\mathrm{P}$ loses, then $\mathrm{P}$ must decide whether to give up or to litigate (through a judicial court). If $\mathrm{P}$ wins, then G must decide whether to remove the links, litigate, or be in default of the removal order of the dataprotection authority; if $\mathrm{G}$ defaults, then $\mathrm{P}$ again has to decide whether to litigate.

${ }^{22}$ The market might also form the expectation that many links will be removed from Google, which would cast doubt on Google's function as a search engine and subsequently cause its user base to shrink.

${ }^{23}$ If the search engine incurs an additional loss, say $d>0$, only when the petitioner wins the further verdict, then the expected payoff for the search engine should be written $-\beta(l+d)-C_{\mathrm{G}}$ instead of internalizing the loss $d$ in $C_{\mathrm{G}}$. In that case, we can allow for an increase in $d$ when the RTBF expands globally, but the comparative statics on the equilibrium probability of link removal with respect to $d$ is effectively the same as that with respect to $l$. 
wake of landmark cases of public interest, which voids the original purpose of trying to protect privacy by removing links pertinent to his identity (see Teffer 2015). Parameter $C_{\mathrm{p}}$ can then incorporate any additional losses to the petitioner from involvement in costly legal proceedings and public exposure.

By interpreting the parameters $C_{\mathrm{G}}$ and $C_{\mathrm{P}}$ more broadly to incorporate the idea of subsequent actions or many petitioners, our simplified model can capture the salient features of the underlying conflict over the RTBF. Our question then reduces to how the changes in $C_{\mathrm{G}}$ and $C_{\mathrm{P}}$ affect the probability of link removal. The formal analysis of this comparative statics leaves our main message qualitatively intact.

\section{Welfare Implications for the Global Expansion}

The incidence of link removals is clearly one measure of interest to the relevant parties of the global expansion debate. To assess the desirability of global expansion, we must also account for another indispensable component that frames the debate, namely, social costs. This consideration allows us to examine the welfare properties of the global expansion: whether extending the domains on which the links are removed brings improvement in social welfare.

Besides the search engine's loss, the removal of links may impose negative externalities on its users. Some Internet users might need to exert more effort or might even fail to find the content they are looking for without the links offered by the search engine that could otherwise help them. ${ }^{24}$ We denote $S \geq 0$ as the expected loss to the search engine's users as a result of deleted links. This parameter $S$ can also include social costs borne by relevant third parties other than the individual users of the search engine. Then $S$ can be broadly interpreted as the interest of the general public in having easy access to the information in question on searching a data subject's name and in having the right to know and to provide information.

We define social welfare as the total payoffs of the petitioner, the search engine, and the relevant third parties (henceforth, users), less fixed costs. ${ }^{25}$ For a given link removal claim represented by $h$, social welfare is equal to $-(1-q) h-l-S$ if the links are removed and $-h$ if the links are not removed. Let $\operatorname{Pr}(q, l ; h)$ denote the equilibrium probability of link removal for a claim associated with harm $h$ if the petitioner recovers $q$ of his harm and the search engine loses $l$ from the removal. Then the ex ante expected social welfare, denoted $W(q, l, S ; h)$, is

$$
\begin{aligned}
W(q, l, S ; h) & =\operatorname{Pr}(q, l ; h)[-(1-q) h-l-S]+[1-\operatorname{Pr}(q, l ; h)](-h) \\
& =\{-h+[\operatorname{Pr}(q, l ; h)] q h\}+[\operatorname{Pr}(q, l ; h)(-1-S)],
\end{aligned}
$$

\footnotetext{
${ }^{24}$ One might expect that removing links could reduce noise, which would make it easier to find other information. In contrast, Eric Posner argues that the RTBF raises the cost for someone to find contested information by making it inconvenient (see Laursen 2015).

${ }^{25}$ By fixed costs, we mean the claim and appeal costs. Their exclusion in the computation of social welfare does not drive the results; rather, it obviates a trivial argument that social welfare is maximized under a no-claim equilibrium when costs are high enough.
} 
which depends on the scope of link removal. In this expression, the first component $U(q, l ; h) \equiv\{-h+[\operatorname{Pr}(q, l ; h)] q h\}$ represents the ex ante welfare of the petitioner requesting link removal. ${ }^{26}$ The second component $V(q, l, S ; h) \equiv[\operatorname{Pr}(q, l ; h)$ $(-l-S)]$ represents the ex ante welfare of the search engine and the users from the removal request.

To investigate the welfare effects of global expansion, we need to take into account a probable change in the users' loss that would result. While the search engine's users can circumvent the local deletion of links by using a device with a non-European Internet protocol address, expanding the scope of deletion further hinders the users' easy access to information. Investors and tech entrepreneurs would expect higher costs of doing business and of providing information via the search engine for which the RTBF applies globally. Hence, in a vein similar to assumption 1, we can reasonably expect that the users' loss from the resulting removal will be larger if the removal of the links is applied in a global manner.

Assumption 2. The global expansion of the RTBF induces an increase in $S$.

Let $l$ and $S$ be the values of losses for the search engine and the users, respectively, under the limited link removal for which the petitioner recovers $q<1$ of his harm $h$, and let $l^{\prime}$ and $S^{\prime}$ be the increased losses to the search engine and the users, respectively, under the extended link removal for which the petitioner recoups the entirety $q=1$ of his harm. Then the global expansion improves social welfare if and only if $W\left(1, l^{\prime}, S^{\prime} ; h\right) \geq W(q, l, S ; h)$ or, equivalently,

$$
U\left(1, l^{\prime} ; h\right)+V\left(1, l^{\prime}, S^{\prime} ; h\right) \geq U(q, l ; h)+V(q, l, s ; h) .
$$

If $\operatorname{Pr}\left(1, l^{\prime} ; h\right) \geq \operatorname{Pr}(q, l ; h)$, then $U\left(1, l^{\prime} ; h\right)>U(q, l ; h)$ and $V\left(1, l^{\prime}, S^{\prime} ; h\right)<V(q, l$, $S ; h)$, but whether inequality (4) holds is ambiguous. ${ }^{27}$ If $\operatorname{Pr}\left(1, l^{\prime} ; h\right)<\operatorname{Pr}(q, l ; h)$, then whether $U\left(1, l^{\prime} ; h\right) \gtrless U(q, l ; h)$ and $V\left(1, l^{\prime}, S^{\prime} ; h\right) \gtrless V(q, l, s ; h)$ are both unclear, because they crucially rely on the underlying parameters. This discussion proves the following proposition.

Proposition 4. Under assumptions 1 and 2, for a given removal claim,

i) if the probability of link removal increases because of the expansion, then the global expansion is necessarily ex ante welfare improving for the petitioner but welfare decreasing for the search engine and the users;

ii) if the probability of link removal decreases because of the expansion, then the global expansion might increase or decrease the ex ante welfare of the petitioner or that of the search engine and the users.

In either case, the global expansion may either enhance or reduce the ex ante social welfare.

\footnotetext{
${ }^{26}$ Given the equilibrium probability of link removal $\operatorname{Pr}(q, l ; h)$, the petitioner can assign an expected value to the subgame following his claim: $U(q, l ; h)=[\operatorname{Pr}(q, l ; h)][-(1-q) h]+[1-\operatorname{Pr}(q$, $l ; h)](-h)$.

${ }^{27}$ Even when $\operatorname{Pr}\left(1, l^{\prime} ; h\right)=\operatorname{Pr}(q, l ; h)$, inequality (4) holds if and only if $(1-q) h \geq\left(l^{\prime}-l\right)+$ $\left(S^{\prime}-S\right)$.
} 
The reasoning behind proposition 4.i is straightforward because if global expansion results in a higher probability of link removal, then the petitioner would recover more while the search engine and the users would lose more. It may seem surprising in proposition 4.ii that the petitioner's welfare can still improve or that the counterpart's welfare can be reduced even with a lower probability of link removal. If the level of petitioner's harm that is salvageable by limited removal is low, then the additional harm that he can recover by global removal will be large, in which case the petitioner can improve ex ante welfare. ${ }^{28}$ Likewise, global expansion can generate comparable losses for the search engine and the users so that their ex ante welfare decreases. ${ }^{29}$

Proposition 4 shows that the welfare effects of the global expansion on the petitioner and on the search engine and its users are ambiguous and that the effects on the two counterparts are not always in opposition. We can consider the welfare for the petitioner and that for the search engine and its users as the measures that quantify the extent to which privacy and freedom of speech, respectively, are strengthened. In this sense, if the global expansion brings about more link removals at the margin, only then we can say that it necessarily reinforces privacy but undermines free speech. However, it is not clear whether global expansion generates more link removals at the margin (see corollary 1). When it does not, global expansion can be undesirable for privacy but desirable for free speech or privacy and free speech need not be in conflict.

The rights to privacy and free speech may generally represent two conflicting interests. But our analysis of the welfare effects implies that the debate over the RTBF expansion should not immediately be seen as promoting privacy while endangering free speech in terms of the welfare of the relevant parties. Further, the global expansion of the RTBF may bring either greater or lesser social welfare. Whether it is socially desirable depends on many factors. ${ }^{30}$ Roughly put, the greater the petitioner's salvageable harm from the limited deletion and the larger the increase in the search engine's and users' losses by the expansion, the more

\footnotetext{
${ }^{28}$ The following numerical example illustrates this point. Suppose that $h=50, q=.35$, and $l=$ 10 under the limited scope of removal. (The parameters can be interpreted in dollars. Assume that the appeal costs for both players are 5 and that $\beta$ is uniformly distributed.) Then $\operatorname{Pr}(.35,10 ; 50)=$ 58.1 percent and $U(.35,10 ; 50)=-39.83$. If global removal renders $q=1$ and $l^{\prime}=15$, then $\operatorname{Pr}(1$, $15 ; 50)=55.6$ percent, and the petitioner's ex ante welfare increases to $U(1,15 ; 50)=-22.22$. If $l^{\prime}$ $=25$, then $\operatorname{Pr}(1,25 ; 50)=52$ percent, and the petitioner's ex ante welfare still increases to $U(1,25$; $50)=-24$. Even if the petitioner can recover a lot from limited removal, for example, $q=.85$, so $\operatorname{Pr}(.85,10 ; 50)=62.5$ percent and $U(.85,10 ; 50)=-23.44$, the petitioner's ex ante welfare increases if $l^{\prime}=15$ (but decreases if $l^{\prime}=25$ ).

${ }^{29}$ Continuing from the previous example, let us suppose that the users' loss increases from $S=$ 50 to $S^{\prime}=60$ because of the expansion. Then the ex ante welfare for the search engine and users decreases from $V(.35,10,50 ; 50)=-34.86$ to $V(1,15,60 ; 50)=-41.67$. The changes in the losses are arbitrary, but this example illustrates that the ex ante welfare for these parties can decrease even with a lower expected probability of link removal.

${ }^{30}$ The factors include the size of relevant parameter values, the magnitude of changes in parameter values because of global expansion, and both the magnitude and the direction of a change in the probability of link removal because of global expansion. Further, there are many claims by petitioners with different levels of harm and salvageable proportion, for which the losses to the search engine and users also vary.
} 
likely it is that the global expansion decreases the possibility of link removals and lowers social welfare.

\section{Concluding Remarks}

There will be continued debate over the expansion of the RTBF. The consensus among many scholars and practitioners in the debate is that the expansion will increase the incidence of link removals and hence increase privacy protections. However, the discussions miss an important part of the underlying economics: the expansion can change the relevant parties' payoffs associated with link removals. We identify those fundamental forces and assess the impact of the expansion of the RTBF on link removals.

Our analysis has interesting implications for the current debate over expanding the scope of the RTBF. We show that the global expansion can decrease link removals at the margin. Hence, the expansion can unexpectedly have negative consequences for privacy but positive consequences for free speech in terms of the incidence of link removals. If we evaluate the expansion in terms of the welfare of individuals who request link removals and those who seek information through search results, global expansion can support individuals' privacy without hampering freedom of speech and open access to information. The benefit of our analysis is that it offers a theoretical framework that is tractable yet flexible enough to handle several nuanced considerations that surround the tension between privacy and free speech in the current debate. This paper shows that global expansion is not necessarily a reinforcement of privacy nor a threat to freedom of speech on the basis of either link removals or efficiency; hence, it advocates a reformulation of the debate on the global expansion of the RTBF.

Several directions for future research are worth considering, particularly those related to third parties' losses due to link removal. The parameter $S$ does not directly enter into the payoffs to the two players in our RTBF game. In the evaluation process of link removals by a search engine or an authority such as the DPA or judicial court, the decision about removal depends on factual issues relevant to the links in question. ${ }^{31}$ Those factual issues consist of comparing the petitioner's harm to both the search engine's and the users' loss. The parameter $\beta$ in our model describes the expected ruling of the DPA, but we do not impose any restrictions on how $\beta$ might depend on the factual issues except that the search engine has a better estimate of $\beta$. A natural next step for future research would be to extend the analysis of the effects of the global expansion in a game with $\beta$ that depends on the relevant parameters, including $S$.

Another interesting avenue for future research is to consider the social costs of the RTBF induced by the distortion in information due to link removal. The

\footnotetext{
${ }^{31}$ For example, the judgment of the European Court of Justice in the Costeja case was based on the grounds that the data subject's fundamental rights to the protection of personal information and to privacy override "not only the economic interest of the operator of the search engine but also the interest of the general public in having access to that information" (Case C-131/12, 2014 E.C.J. 317, para. 99).
} 
RTBF laws might help protect the dignity of an individual who is continuously tarnished by past wrongful behavior by making erasure from the never-forgetting Internet easier, which thus offers a clean slate to the individual. ${ }^{32}$ However, the erasure of names of people with past unfavorable behavior from search results can pose considerable threats to reputational capital (Mcelroy 2014). Employers obtain recommendation letters about potential employees; business thrives with a good reputation and withers with a bad one. The reputation systems are vital in various markets and networks, but RTBF laws might create biased reputational capital of some individual or entity, distort the system users' inference and the entity's incentives and behaviors, and hinder matching benefits, which would create large welfare losses for the general public (that can be captured by $S$ ).$^{33}$ It will be interesting and worthwhile to investigate the negative externality effects of link removals and global expansion on the system of reputational capital for future research.

\section{Appendix}

\section{Proofs}

\section{A1. Preliminaries}

We represent the petitioner's strategy by $\left(p_{c}, p_{\mathrm{a}}\right)$, where the first component indicates P's probability of claiming and the second is his conditional probability of appealing if the claim is rejected. We begin by deriving condition $2\left(l>C_{\mathrm{G}}\right)$. We want $\beta_{\mathrm{G}}>0$ to have some types of $\mathrm{G}$ reject. Because $\beta_{\mathrm{G}}=\left(l / p_{\mathrm{a}}-C_{\mathrm{G}}\right) / l$ is decreasing in $p_{\mathrm{a}}$, it suffices to have $\beta_{\mathrm{G}}>0$ at $p_{\mathrm{a}}=1$. Then $l>C_{\mathrm{G}}$ if and only if $\beta_{\mathrm{G}}^{\star} \equiv\left(l-C_{\mathrm{G}}\right) / l>0$, which implies that $\beta_{\mathrm{G}}>0$ for any given $p_{\mathrm{a}}>0$.

Lemma A1. Petitioner P's best response to rejection by Gs of type $\beta \leq \beta_{\mathrm{G}}$ is

i) if $\beta_{\mathrm{G}}>\beta^{*}$, then $p_{\mathrm{a}}=1$;

ii) if $\beta_{\mathrm{G}}=\beta^{*}$, then $p_{\mathrm{a}} \in[0,1]$;

iii) if $\beta_{\mathrm{G}}<\beta^{*}$, then $p_{\mathrm{a}}=0$.

Proof. Petitioner P's expected payoff from an appeal (if the claim is rejected by Gs of type $\beta \leq \beta_{\mathrm{G}}$ ) depends on the posterior expectation of $\beta$ on the interval $\left[0, \beta_{\mathrm{G}}\right]$. If $\beta_{\mathrm{G}}$ increases, then the expected probability of P's winning $\mathbb{E}\left[\beta \mid \beta \leq \beta_{\mathrm{G}}\right]$ increases, so the expected value of appealing increases. By definition of $\beta^{\star}$, the posterior expectation of $\beta$ with beliefs con-

\footnotetext{
${ }^{32}$ Employers use criminal history records in the employment screening process (Bushway 2004). First-time criminal conviction reduces employment probabilities and income, so individuals with conviction records are more likely to experience job instability (Waldfogel 1994; Nagin and Waldfogel 1995). However, Blumstein and Nakamura (2009) find that a person with a criminal record who remains free of additional offenses for a certain period of time provides no greater risk than a nonoffender. Therefore, privacy protection through limiting easy access to criminal history might encourage nondiscrimination in employment and investment in rehabilitation.

${ }^{33}$ As a related point, some argue that the global expansion of the European RTBF might lead to more censorship by public officials such as autocrats who want to whitewash the past or remove links they do not like. See New York Times (2015).
} 
centrated on $\left[0, \beta^{*}\right]$ leads to $\mathbb{E}\left[\beta \mid \beta \leq \beta^{*}\right] q h=C_{\mathrm{p}}$. For the case in lemma A1.i, if $\beta_{\mathrm{G}}>\beta^{*}$, then $\mathbb{E}\left[\beta \mid \beta \leq \beta_{\mathrm{G}}\right] q h>\mathbb{E}\left[\beta \mid \beta \leq \beta^{*}\right] q h=C_{\mathrm{P}}$. So P must always appeal; that is, $p_{\mathrm{a}}=1$. For the case in lemma Al.ii, if $\beta_{\mathrm{G}}=\beta^{*}$, then $\mathbb{E}\left[\beta \mid \beta \leq \beta_{\mathrm{G}}\right] q h=\mathbb{E}\left[\beta \mid \beta \leq \beta^{\star}\right] q h=C_{\mathrm{p}}$. Then $\mathrm{P}$ is indifferent between appealing and giving up, so $\mathrm{P}$ follows a randomized strategy $p_{\mathrm{a}} \in[0,1]$. For the case in lemma A1.iii, if $\beta_{\mathrm{G}}<\beta^{\star}$, then $\mathbb{E}\left[\beta \mid \beta \leq \beta_{\mathrm{G}}\right] q h<\mathbb{E}\left[\beta \mid \beta \leq \beta^{\star}\right] q h=C_{\mathrm{P}}$. Hence, it must be that $p_{\mathrm{a}}=0$. Q.E.D.

\section{A2. Proof of Proposition 1}

Consider the subgame following the claim. Under condition 2 that ensures that $\beta_{\mathrm{G}}>0, \mathrm{P}$ uses Bayes's theorem to compute his posterior expections of G's type given the prior expectations when the claim is rejected.

For the case in proposition 1.i, it is immediately obvious that $\beta_{\mathrm{G}}^{*} \equiv$ $\left(l-C_{\mathrm{G}}\right) / l \leq\left(l / p_{\mathrm{a}}-C_{\mathrm{G}}\right) / l=\beta_{\mathrm{G}}$ for any $p_{\mathrm{a}}>0$ because $\beta_{\mathrm{G}}$ is decreasing in $p_{\mathrm{a}} \leq 1$. Therefore, if $\beta_{\mathrm{G}}^{*}>\beta^{*}$, then $\beta_{\mathrm{G}}>\beta^{*}$ for any $p_{\mathrm{a}}>0$. Then P's best-response strategy must be $p_{\mathrm{a}}=1$ by lemma A1. Against P's strategy $p_{\mathrm{a}}=1$, G's best response is to use the cutoff strategy with the cutoff type $\beta_{\mathrm{G}}$ that equals $\beta_{\mathrm{G}}^{*}$. Hence, Gs of type $\beta \leq \beta_{\mathrm{G}}^{*}$ reject the claim, and all others accept it, believing that $\mathrm{P}$ will appeal with a probability of 1 . This in turn justifies P's optimal strategy to be $p_{a}=1$. These strategies of $\mathrm{G}$ and $\mathrm{P}$ constitute the only subgame-perfect Nash equilibrium after P's claim.

For the case in proposition 1.ii, if $\beta_{\mathrm{G}}^{\star} \leq \beta^{*}$, then $\beta_{\mathrm{G}} \gtreqless \beta^{*}$ depends on P's strategy $p_{\mathrm{a}}$. First suppose that $p_{\mathrm{a}}=0$. Then all Gs reject the claim because G expects $P$ to definitely give up, and $G$ earns 0 by rejecting instead of $-l$ by accepting. At rejection by all Gs, P learns nothing additional about G's type, which implies that his posterior expectation of $\beta$ equals his prior expectation; however by condition $1, \mathrm{P}$ will prefer appealing to giving up, so $p_{\mathrm{a}}=1$, which is a contradiction. Now suppose that $p_{\mathrm{a}}=1$. Then $\beta_{\mathrm{G}}=\beta_{\mathrm{G}}^{*}\left(\leq \beta^{*}\right)$. If $\beta_{\mathrm{G}}<\beta^{*}$, then $p_{\mathrm{a}}$ must equal 0 by lemma A1, which again leads to a contradiction. Therefore, it must be the case that $\beta_{\mathrm{G}}=\beta_{\mathrm{G}}^{*}=\beta^{*}$. Note that $p_{\mathrm{a}}$ can be computed using $\beta_{\mathrm{G}}=\left(l / p_{\mathrm{a}}-C_{\mathrm{G}}\right) / l$. That is, $p_{\mathrm{a}}=l /\left(\beta_{\mathrm{G}} l+C_{\mathrm{G}}\right)=l /\left(\beta^{\star} l+C_{\mathrm{G}}\right)=l /\left(\beta_{\mathrm{G}}^{\star} l+C_{\mathrm{G}}\right)=1$, where the last equality follows from the definition of $\beta_{\mathrm{G}}^{*}=\left(l-C_{\mathrm{G}}\right) / l$. This confirms P's strategy to appeal with a probability of 1 . Last, if $p_{\mathrm{a}} \in(0,1)$, then it must be the case that $\beta_{\mathrm{G}}=\beta^{*}$ by lemma A1. Given G's cutoff strategy, P is indifferent between appealing and giving up because $\mathbb{E}\left[\beta \mid \beta \leq \beta^{*}\right] q h=C_{\mathrm{P}}$, which in turn justifies that $\mathrm{P}$ uses a randomized strategy $p_{\mathrm{a}} \in(0,1)$. For $\beta^{\star}$ to be G's optimal cutoff type, we must have $l=p_{\mathrm{a}}\left(\beta^{*} l+C_{\mathrm{G}}\right)$, which implies that $p_{\mathrm{a}}$ is uniquely determined by

$$
p_{\mathrm{a}}=\frac{l}{\beta^{\star} l+C_{\mathrm{G}}} .
$$

Therefore, believing that $\mathrm{P}$ randomizes between appealing and giving up with the probability given in equation (A1), G's best response is to use the cutoff type $\beta_{\mathrm{G}}$ 
$=\beta^{*}$. Thus, if $\beta_{\mathrm{G}}^{*} \leq \beta^{*}$, then G's cutoff strategy given by $\beta_{\mathrm{G}}=\beta^{*}$ and P's strategy $p_{\mathrm{a}}$ given by equation (A1), where $p_{\mathrm{a}}=1$ if and only if $\beta^{*}=\beta_{\mathrm{G}}^{*}$, constitute the only subgame-perfect Nash equilibrium following the claim.

The equilibrium strategies described above for each case form the unique equilibrium in behavioral strategies, where the beliefs are weakly consistent. Because $F\left(\beta_{\mathrm{G}}\right)>0$ by condition 2 , the rejection state is never a zero-probability event. Thus, weak consistency implies full consistency of beliefs with the unique equilibrium in behavioral strategies. Q.E.D.

\section{A3. Proof of Proposition 2}

At P's initial node, his no-claim payoff is $-h$, and his expected payoff from the claim is obtained under the prior distribution of G's types given the subgame equilibrium strategies as follows:

$$
\begin{gathered}
{\left[1-F\left(\beta_{\mathrm{G}}\right)\right][-(1-q) h-c]} \\
+F\left(\beta_{\mathrm{G}}\right)\left[\left(1-p_{\mathrm{a}}\right)(-h-c)+p_{\mathrm{a}}\left(-\left[1-\mathbb{E}\left[\beta \mid \beta \leq \beta_{\mathrm{G}}\right] q\right] h-c-C_{\mathrm{P}}\right)\right] .
\end{gathered}
$$

Then P's optimal strategy at his initial node is to claim if the value in expression (A2) is greater than or equal to $-h$. This condition can be written as expression (1).

If $\beta_{\mathrm{G}}^{*}>\beta^{\star}$, then $\beta_{\mathrm{G}}=\beta_{\mathrm{G}}^{*}$ and $p_{\mathrm{a}}=1$ form a unique equilibrium in the subgame where P's posterior expectation of G's type is given by $\mathbb{E}\left[\beta \mid \beta \leq \beta_{\mathrm{G}}^{*}\right]$. Expression (1) then becomes

$$
c \leq\left[1-F\left(\beta_{\mathrm{G}}^{*}\right)\right] q h+F\left(\beta_{\mathrm{G}}^{*}\right)\left[\mathbb{E}\left[\beta \mid \beta \leq \beta_{\mathrm{G}}^{*}\right] q h-C_{\mathrm{P}}\right] .
$$

If $c$ is such that inequality (A3) holds, then $\mathrm{P}$ always prefers claim to no claim. Therefore, P's strategy profile $\left(p_{\mathrm{c}}, p_{\mathrm{a}}\right)=(1,1)$, G's cutoff strategy with $\beta_{\mathrm{G}}=\beta_{\mathrm{G}}^{*}$, and P's posterior expectation $\mathbb{E}\left[\beta \mid \beta \leq \beta_{\mathrm{G}}^{*}\right]$ at rejection form a unique sequential equilibrium of this game. If $c$ is larger than the right-hand side of inequality (A3), then $\left(p_{c}, p_{\mathrm{a}}\right)=(0,1), \beta_{\mathrm{G}}=\beta_{\mathrm{G}}^{*}$, and P's posterior expectation $\mathbb{E}\left[\beta \mid \beta \leq \beta_{\mathrm{G}}^{*}\right]$ at rejection form a sequential equilibrium. The sequential equilibrium is unique; otherwise, there must be multiple subgame-perfect equilibria, which would contradict the uniqueness of the Nash equilibrium in the subgame specified in proposition 1 .

If $\beta_{\mathrm{G}}^{*} \leq \beta^{*}$, then given the subgame equilibrium specified in proposition 1, expression (1) becomes $c \leq\left[1-F\left(\beta^{\star}\right)\right] q h$ because $\left[\mathbb{E}\left[\beta \mid \beta \leq \beta^{\star}\right] q h-C_{\mathrm{P}}\right]=0$ and $\beta_{\mathrm{G}}=\beta^{\star}$. Then the same argument as in the previous case of $\beta_{\mathrm{G}}^{*}>\beta^{\star}$ proves that there is a unique sequential equilibrium in which $p_{\mathrm{c}}=1$ if $c \leq\left[1-F\left(\beta^{\star}\right)\right]$ $q h$ and $p_{\mathrm{c}}=0$ otherwise. Q.E.D.

\section{A4. Equilibrium Characterization when Condition 1 or 2 Fails}

Proposition A1. When condition 1 fails, there is a unique sequential equilibrium of the RTBF game in which $p_{\mathrm{c}}=0$, all Gs reject, and $p_{\mathrm{a}}=0$. When condi- 
tion 2 fails while condition 1 holds, there are two sequential equilibria in which the equilibrium strategies are characterized as follows:

i) $p_{\mathrm{c}}=1$ if $c \leq\left[1-F\left(\beta^{\star}\right)\right] q h$ and 0 otherwise, Gs of type $\beta>\beta^{\star}$ accept and all others reject, and $p_{\mathrm{a}}=l /\left(\beta^{*} l+C_{\mathrm{G}}\right)$;

ii) $p_{\mathrm{c}}=1$ if $c \leq q h$ and 0 otherwise, all Gs accept, and $p_{\mathrm{a}}=1$.

Proof. First, if the parameter values are such that condition 1 is violatedthat is, $\mathbb{E}[\beta] q h<C_{\mathrm{P}}$-then appealing is not profitable given that all Gs reject. If more Gs accept, then $\mathrm{P}$ lowers his posterior expectation of the probability of winning; hence, appealing becomes even less profitable (that is, $\mathbb{E}\left[\beta \mid \beta \leq \beta_{\mathrm{G}}\right] q h<\mathbb{E}[\beta] q h<C_{\mathrm{P}}$ for any $\left.\beta_{\mathrm{G}}<1\right)$. This implies that only $p_{\mathrm{a}}=$ 0 is sequentially rational given $\mathrm{P}$ 's posterior beliefs. Hence, there is a unique subgame-perfect equilibrium following P's claim: all Gs reject; after rejection, P's posterior equals his prior, and therefore $\mathrm{P}$ chooses to give up; thus, $\mathrm{G}$ was correct to reject the claim. Regardless of whether condition 2 holds, this is the only subgame equilibrium in which the strategies are sequentially rational. Now given such equilibrium strategies in the subgame, P's expected payoff from the claim in expression (A2) becomes $-h-c$, whereas his no-claim payoff is $-h$, so $\mathrm{P}$ will choose to not claim. Hence, if condition 1 fails, then there is a unique sequential equilibrium in which $\mathrm{P}$ never claims, all Gs reject, and $\mathrm{P}$ always gives up.

Second, suppose that the parameter values are such that condition 2 is violated-that is, $l \leq C_{\mathrm{G}}$-while condition 1 holds. If $p_{\mathrm{a}}=0$, then all Gs will reject; on rejection, $\mathrm{P}$ will prefer appealing to giving up according to condition 1 , which is a contradiction. If $\mathrm{G}$ believes that $\mathrm{P}$ will appeal with some positive probability $p_{\mathrm{a}}<1$, then it must be the case that $\beta_{\mathrm{G}}=\beta^{*}$, and thus $l=p_{\mathrm{a}}\left(\beta^{*} l+C_{\mathrm{G}}\right)$, or equivalently $p_{\mathrm{a}}=l /\left(\beta^{*} l+C_{\mathrm{G}}\right)$. This $p_{\mathrm{a}}$ satisfies $-l \leq p_{\mathrm{a}}\left[-\beta l-C_{\mathrm{G}}\right]$ for any $\beta \leq$ $\beta^{\star}$. These strategies of $\mathrm{G}$ and $\mathrm{P}$ are equivalent to those in proposition 1.ii, and so an argument identical to that in the proof of proposition 2 proves a sequential equilibrium. If $G$ believes that $P$ will appeal with a probability of 1 , then $G$ of any type prefers to accept. This is because $-l \geq-\beta l-C_{\mathrm{G}}$ for all $\beta \in[0,1]$, where the equality holds if and only if $l=C_{\mathrm{G}}$ and $\beta=0$, which happens with a probability of 0 . Hence, rejection is a zero-probability event, in which case $\mathrm{P}$ is free to use a posterior belief over $G$ types that are rejecting that is identical to his prior belief over $G$ types in a sequential equilibrium. Condition 1 then justifies P's strategy $p_{\mathrm{a}}=1$, and, thus, $\mathrm{G}$ was correct to accept the claim regardless of her type. Given such equilibrium strategies in the subgame, expression (1) reduces to $c \leq q h$. Therefore, strategies in which $\mathrm{P}$ claims if $c \leq q h$ and does not claim otherwise, all Gs accept, and $\mathrm{P}$ always appeals form a second sequential equilibrium of the game. Q.E.D.

\section{A6. Proof of Proposition 3}

We begin by describing how the best responses of $\mathrm{G}$ characterized by the cutoff type $\beta^{\star}$ or $\beta_{\mathrm{G}}^{\star}$ vary with the proportion of $\mathrm{P}^{\prime}$ 's harm recovered from removal 
(q) or with the amount of G's loss (l). These comparative statics results are easily obtained from observing the definitions of $\beta^{*}$ and $\beta_{\mathrm{G}}^{*}$, summarized as follows:

$$
\frac{d \beta^{\star}}{d q}<0, \quad \frac{d \beta_{\mathrm{G}}^{\star}}{d q}=0 ; \quad \frac{d \beta^{\star}}{d l}=0, \quad \frac{d \beta_{\mathrm{G}}^{*}}{d l}>0 .
$$

When $\beta_{\mathrm{G}}^{*} \leq \beta^{\star}$, G's optimal cutoff type is $\beta^{*}$, which is defined by $\mathbb{E}[\beta \mid \beta \leq$ $\left.\beta^{*}\right] q h=C_{\mathrm{P}}$, so on rejection by Gs of type $\beta \leq \beta^{*}$, $\mathrm{P}$ is indifferent between giving up and appealing. A higher $q$ leads to a lower cutoff type $\beta^{*}$, whereas $\beta^{*}$ is not affected by the amount of G's loss $l$. When $\beta_{\mathrm{G}}^{*}>\beta^{*}$, G's optimal cutoff type is $\beta_{\mathrm{G}}^{*}=\left(l-C_{\mathrm{G}}\right) / l$, which is not affected by $q$. On the other hand, a higher $l$ increases $\beta_{\mathrm{G}}^{*}$.

The probability of link removal, $\operatorname{Pr}($ Link Removal $) \equiv\left[1-F\left(\beta_{\mathrm{G}}\right)\right]+$ $F\left(\beta_{\mathrm{G}}\right) p_{\mathrm{a}} \mathbb{E}\left[\beta \mid \beta \leq \beta_{\mathrm{G}}\right]$, can be rewritten as

$$
\operatorname{Pr}(\text { Link Removal })=1-\left(1-p_{\mathrm{a}} \beta_{\mathrm{G}}\right) F\left(\beta_{\mathrm{G}}\right)-p_{\mathrm{a}} \int_{0}^{\beta_{\mathrm{G}}} F(x) d x
$$

because $\mathbb{E}\left[\beta \mid \beta \leq \beta_{\mathrm{G}}\right]=\int_{0}^{\beta_{\mathrm{G}}}\left[x f(x) / F\left(\beta_{\mathrm{G}}\right)\right] d x=\beta_{\mathrm{G}}-\left[1 / F\left(\beta_{\mathrm{G}}\right)\right] \int_{0}^{\beta_{\mathrm{G}}} F(x) d x$ by partial integration.

\section{Proof of Proposition 3.i}

First consider the case of $q \in\left[q, q^{*}\right)$, where we have $p_{\mathrm{a}}=l /\left(\beta^{*} l+C_{\mathrm{G}}\right) \in(0,1)$ and $\beta_{\mathrm{G}}=\beta^{*}<1$ in equilibrium. Then differentiation of expression (A5) with respect to $q$ shows that the probability of link removal unambiguously increases with a small increase in $q$ :

$$
\begin{aligned}
\frac{d \operatorname{Pr}(\text { Link Removal })}{d q} & =\left\{\frac{\partial\left[1-\left(1-p_{\mathrm{a}} \beta^{*}\right) F\left(\beta^{*}\right)-p_{\mathrm{a}} \int_{0}^{\beta^{*}} F(x) d x\right]}{\partial \beta^{*}}\right] \frac{d \beta^{*}}{d q} \\
& =\left[-\left(1-p_{\mathrm{a}} \beta^{*}\right) f\left(\beta^{*}\right)-F\left(\beta^{*}\right)\left[-p_{\mathrm{a}}+\beta^{*}\left(p_{\mathrm{a}}\right)^{2}\right]+\left(p_{\mathrm{a}}\right)^{2} \int_{0}^{\beta^{*}} F(x) d x-p_{\mathrm{a}} F\left(\beta^{*}\right)\right] \frac{d \beta^{*}}{d q} \\
& =\underbrace{\left[-\left(1-p_{\mathrm{a}} \beta^{*}\right) f\left(\beta^{*}\right)-F\left(\beta^{*}\right)\left(p_{\mathrm{a}}\right)^{2}\left(\beta^{*}-\frac{1}{F\left(\beta^{*}\right)} \int_{0}^{\beta^{*}} F(x) d x\right)\right]}_{<0} \underbrace{\frac{d \beta^{*}}{d q}>0,}_{<0}
\end{aligned}
$$

where the second equality uses $\partial p_{\mathrm{a}} / \partial \beta^{*}=-\left(p_{\mathrm{a}}\right)^{2}$ and $d\left[\int_{0}^{\beta_{\mathrm{G}}} F(x) d x\right] / d \beta_{\mathrm{G}}=$ $F\left(\beta_{\mathrm{G}}\right)$ by the Leibniz integral rule. Noticing that $\left\{\beta^{\star}-\left[1 / F\left(\beta^{\star}\right)\right] \int_{0}^{\beta^{*}} F(x) d x\right\}=$ $\mathbb{E}\left[\beta \mid \beta \leq \beta^{\star}\right]$, it is clear that the term in brackets in the third line is negative. Next consider the case $q \geq q^{*}$ for which $p_{\mathrm{a}}=1$ and $\beta_{G}=\beta_{\mathrm{G}}^{*}=\left(l-C_{G}\right) / l$ in equilibrium. These equilibrium strategies are not affected by an increase in $q$ for $q$ $\in\left[q^{*}, 1\right)$, where the boundary condition will continue to hold for a small increase in $q$. Hence, an increase in $q$ leads to exactly the same probability of link removal when $q \geq q^{*}$. 


\section{Proof of Proposition 3.ii}

First consider the case of $l \in\left(C_{\mathrm{G}}, l^{*}\right)$. (That $C_{\mathrm{G}}$ is the lower bound of $l$ is implied by condition 2.) Because $l<l^{\star}$ is in strict inequality, $l<l^{*}$ continues to hold for a small increase in $l$. When $l \in\left(C_{\mathrm{G}}, l^{*}\right)$, we have $p_{\mathrm{a}}=l /\left(\beta^{*} l+C_{\mathrm{G}}\right)$ and $\beta_{\mathrm{G}}=\beta^{*}$ in equilibrium. Then we can rewrite the probability of link removal as $\left[1-F\left(\beta^{\star}\right)\right]+F\left(\beta^{\star}\right) p_{\mathrm{a}} \mathbb{E}\left[\beta \mid \beta \leq \beta^{\star}\right]$. The probability of $\mathrm{G}^{\prime}$ s acceptance, $1-$ $F\left(\beta^{*}\right)$; the probability of $\mathrm{G}^{\prime}$ 's rejection, $F\left(\beta^{\star}\right)$; and the posterior expectation of $\beta$, $\mathbb{E}\left[\beta \mid \beta \leq \beta^{\star}\right]$, are not affected by an increase in $l$ because $d \beta^{\star} / d l=0$. But the probability that $\mathrm{P}$ appeals on rejection, $p_{\mathrm{a}}=l /\left(\beta^{\star} l+C_{\mathrm{G}}\right)$, increases with $l$. Therefore, a small increase in $l$ unambiguously leads to a higher probability of link removal when $l \in\left(C_{\mathrm{G}}, l^{*}\right)$. Now consider the case of $l \geq l^{*}$ for which $p_{\mathrm{a}}=1$ and $\beta_{\mathrm{G}}=\beta_{\mathrm{G}}^{*}$ in equilibrium. In this case, the probability of link removal (equation [A5]) can be rewritten as

$$
1-\left(1-\beta_{\mathrm{G}}^{*}\right) F\left(\beta_{\mathrm{G}}^{*}\right)-\int_{0}^{\beta^{*}} F(x) d x .
$$

Differentiation of expression (A6) with respect to $l$ yields, using the Leibniz integral rule,

$$
\left[-\left(1-\beta_{\mathrm{G}}^{*}\right) f\left(\beta_{\mathrm{G}}^{*}\right)+F\left(\beta_{\mathrm{G}}^{*}\right)-F\left(\beta_{\mathrm{G}}^{*}\right)\right] \frac{d \beta_{\mathrm{G}}^{*}}{d l},
$$

which is equal to $\left[-\left(1-\beta_{\mathrm{G}}^{*}\right) f\left(\beta_{\mathrm{G}}^{*}\right)\right] d \beta_{\mathrm{G}}^{*} / d l$. This is negative because $\left[-\left(1-\beta_{\mathrm{G}}^{*}\right) f\left(\beta_{\mathrm{G}}^{*}\right)\right]<0$ by $\beta_{\mathrm{G}}^{*}<1$ and $d \beta_{\mathrm{G}}^{*} / d l>0$ by expression set (A4). Thus, an increase in $l$ unambiguously leads to a decreased probability of link removal when $l \geq l^{*}$. There is a kink in the probability of link removal at $l=l^{*}$, where $\beta^{*}=\beta_{\mathrm{G}}^{*}=\left(l-C_{\mathrm{G}}\right) / l$ : the left derivative of $\operatorname{Pr}($ Link Removal) evaluated at $l=l^{*}$ is $F\left(1-C_{\mathrm{G}} / l\right)\left(C_{\mathrm{G}} / l^{2}\right) \mathbb{E}\left[\beta \mid \beta \leq 1-C_{\mathrm{G}} / l\right]>0$, whereas the right derivative is $-f\left(1-C_{\mathrm{G}} / l\right)\left[\left(C_{\mathrm{G}}\right)^{2} / l^{3}\right]<0$. The maximum value of the probability of link removal occurs at the kink. Q.E.D.

\section{References}

Acquisti, Alessandro, Curtis Taylor, and Liad Wagman. 2016. The Economics of Privacy. Lournal of Economic Literature 54:442-92.

Bebchuk, Lucian Ayre. 1984. Litigation and Settlement under Imperfect Information. RAND Journal of Economics 15:404-15.

Blumstein, Alfred, and Kiminori Nakamura. 2009. Redemption in the Presence of Widespread Criminal Background Checks. Criminology 47:327-59.

Bushway, Shawn D. 2004. Labor Market Effects of Permitting Employer Access to Criminal History Records. Journal of Contemporary Criminal Justice 20:276-91.

Fleischer, Peter. 2016. Adapting Our Approach to the European Right to Be Forgotten. Keyword (blog), March 4. https://blog.google/topics/google-europe/adapting-our-approach -to-european-rig/. 
Gilbert, Francoise. 2014. Right to Be Forgotten: Guidelines from WP29. Francoise Gilbert on Privacy, Security, and Cloud Computing (blog), November 26. https://www.francoise gilbert.com/?p=954.

Hermalin, Benjamin E., and Michael L. Katz. 2006. Privacy, Property Rights, and Efficiency: The Economics of Privacy as Secrecy. Quantitative Marketing and Economics 4:209-39.

Kovbasyuk, Sergei, and Giancarlo Spagnolo. 2016. Memory and Markets. Working Paper No. 16/06. Einaudi Institute for Economics and Finance, Rome.

Laursen, Lucas. 2015. How Google Handled a Year of "Right to Be Forgotten" Requests. IEEESpectrum, April 23. https://spectrum.ieee.org/telecom/internet/how-google-handled -a-year-of-right-to-be-forgotten-requests.

Mcelroy, Wendy. 2014. The Internet Memory Hole. Freeman, November 24. https://fee .org/articles/the-internet-memory-hole/.

Munson, Lee. 2015. Google's "Right to Be Forgotten” Appeal: France Says "Non!" Naked Security, September 23. https://nakedsecurity.sophos.com/2015/09/23/googles-right-to -be-forgotten-appeal-france-says-non/.

Nagin, Daniel, and Joel Waldfogel. 1995. The Effects of Criminality and Conviction on the Labor Market Status of Young British Offenders. International Review of Law and Economics 15:109-26.

Nalebuff, Barry. 1987. Credible Pretrial Negotiation. RAND Journal of Economics 18:198210.

New York Times. 2015. Europe's Expanding “Right to Be Forgotten.” February 4, p. A24.

Posner, Richard A. 1978. The Right of Privacy. Georgia Law Review 12:393-422. . 1981. The Economics of Privacy. American Economic Review 71:405-9.

Rawlinson, Kevin. 2015. Google in "Right to Be Forgotten" Talks Regulator. BBC News, May 13. http://www.bbc.com/news/technology-32720944.

Rosen, Jeffrey. 2012. The Right to Be Forgotten. Stanford Law Review Online, February. http://www.stanfordlawreview.org/online/privacy-paradox/right-to-be-forgotten.

Stigler, George J. 1980. An Introduction to Privacy in Economics and Politics. Lournal of Legal Studies 9:623-44.

Teffer, Peter. 2015. Europeans Give Google Final Say on “Right to Be Forgotten.” EUobserver, October 8.

Thom, Cleland. 2015. Google "Right to Be Forgotten" Dispute with Information Commissioner Will Impact News Archives. PressGazette British Journalism Awards, September 10. http://awards.pressgazette.co.uk/2015/09/10/google-right-to-be-forgotten-dispute -with-information-commissioner-will-impact-news-archives/.

Tippmann, Sylvia, and Julia Powles. 2015. Google Accidentally Reveals Data on "Right to Be Forgotten" Requests. Guardian, July 14.

Waldfogel, Joel. 1994. The Effect of Criminal Conviction on Income and the Trust "Reposed in the Workmen." Iournal of Human Resources 29:62-81. 


\title{
Online Appendix for "The Economics of the Right to be Forgotten"
}

\author{
Byung-Cheol Kim* $\quad$ Jin Yeub Kim ${ }^{\dagger}$
}

\section{Appendix B}

\section{The Complete Information RTBF Game}

In this online appendix, we first characterize the equilibrium of the RTBF game under complete information about the expected ruling of a data protection authority. We then discuss the impact of expanding the scope of application of the RTBF in the complete information setting.

\section{B1. Equilibrium under Complete Information}

Suppose that both players estimate the likelihood of $\mathrm{P}$ prevailing on appeal to be $\beta \in$ $[0,1]$, which is common and public knowledge. The construction of perfect subgame equilibria is done by backward induction. We begin by computing an optimal strategy for $\mathrm{P}$ at the final node where he decides whether to give up or appeal. The $\mathrm{P}$ compares his payoff from giving up, $-h-c$, with the payoff from appeal, $-(1-\beta q) h-c-C_{P}$. He will give up if $C_{P}>\beta q h$ and appeal if $C_{P} \leq \beta q h$ (assuming that an indifferent $\mathrm{P}$ appeals).

Now consider the decision node where $\mathrm{G}$ decides whether to accept or reject: If $\mathrm{P}$ gives up after being rejected, G's best response is to reject because the payoff from rejecting is higher than that from accepting; that is, $0>-l$. If $\mathrm{P}$ appeals after being rejected, $\mathrm{G}$ will

${ }^{*}$ Department of Economics, Finance and Legal Studies, University of Alabama, 265 Alston Hall, Tuscaloosa, AL 35487-0224. E-mail: byung-cheol.kim@ua.edu, Webpage: https://sites.google.com/site/byungcheolkim76

${ }^{\dagger}$ Department of Economics, University of Nebraska-Lincoln, 1240 R Street, Lincoln, NE 68588-0489, E-mail: shiningjin@gmail.com, Webpage: https://sites.google.com/site/jinyeubkim 
compare her payoff from accepting, $-l$, with the payoff from rejecting, $-\beta l-C_{G}$; so $\mathrm{G}$ will reject if $(1-\beta) l \geq C_{G}$ and accept if $(1-\beta) l<C_{G}$ (assuming that an indifferent $G$ rejects).

Lastly, consider the initial node where $\mathrm{P}$ decides whether to claim or not: If $\mathrm{G}$ rejects and $\mathrm{P}$ gives up in the subsequent nodes, then $\mathrm{P}$ will not claim because $-h>-h-c$. If $\mathrm{G}$ rejects and $\mathrm{P}$ appeals subsequently, then $\mathrm{P}$ will claim if $c+C_{P} \leq \beta q h$ and not claim if otherwise. If $\mathrm{G}$ accepts, then $\mathrm{P}$ will claim if $c \leq q h$ and not claim if otherwise. Taking into account that the claim cost $c>0$ is expected to be very small (because individuals need only complete an online form and need not pay a monetary fee), we may reasonably assume away the case of $c>q h$ without losing important insights. This elimination rules out an equilibrium in which $\mathrm{P}$ does not claim knowing that $\mathrm{G}$ will accept in anticipation of P's appeal in equilibrium. We henceforth maintain the assumption of $c \leq q h$.

For any given parameter values, there exists a unique subgame perfect equilibrium (SPE) in the RTBF game under complete information. The following describes the players' equilibrium strategies in the unique SPE depending on the parameter values:

(1) $\beta<\frac{C_{P}}{q h}$ : P does not claim, $\mathrm{G}$ rejects, and $\mathrm{P}$ gives up.

(2-i) $\frac{C_{P}}{q h} \leq \beta<\frac{c+C_{P}}{q h}$ and $\beta \leq \frac{l-C_{G}}{l}$ : $\mathrm{P}$ does not claim, $\mathrm{G}$ rejects, and $\mathrm{P}$ appeals.

(2-ii) $\frac{c+C_{P}}{q h} \leq \beta$ and $\beta \leq \frac{l-C_{G}}{l}$ : $\mathrm{P}$ claims, $\mathrm{G}$ rejects, and $\mathrm{P}$ appeals.

(3) $\frac{C_{P}}{q h} \leq \beta$ and $\beta>\frac{l-C_{G}}{l}: \mathrm{P}$ claims, $\mathrm{G}$ accepts, and $\mathrm{P}$ appeals. ${ }^{1}$

Consider two extreme cases where both players know the exact decision of the data protection authority. In the first case, if the players expect that the authority will rule in favor of $\mathrm{G}$ (i.e., $\beta=0$ ), then the unique $\mathrm{SPE}$ is that $\mathrm{P}$ never claims, $\mathrm{G}$ rejects, and $\mathrm{P}$ gives up; this corresponds to case (1) above. In the second case, if the players expect the authority to rule in favor of $\mathrm{P}$ (i.e., $\beta=1$ ), then two sub-cases arise: If $1<\frac{C_{P}}{q h}$, then $\mathrm{P}$ never claims,

\footnotetext{
${ }^{1}$ This is the only case where we use the assumption of $c \leq q h$, which we consider to be a minimal restriction on the parameter values given the real cases that our game tries to capture. If $c>q h$, then the unique $\mathrm{SPE}$ is that $\mathrm{P}$ does not claim, $\mathrm{G}$ accepts, and $\mathrm{P}$ appeals.
} 
$\mathrm{G}$ rejects, and $\mathrm{P}$ appeals, which corresponds to case (1); and if $1 \geq \frac{C_{P}}{q h}$, then $\mathrm{P}$ claims, $\mathrm{G}$ accepts, and $\mathrm{P}$ appeals, which corresponds to case (3). Cases (2-i) and (2-ii) never happen because $\frac{l-C_{G}}{l}$ is always less than one. Therefore, in a symmetric information setting where the authority's expected decision is either in favor of $\mathrm{G}$ or in favor of $\mathrm{P}$, the equilibrium involves $\mathrm{P}$ requesting removal only when both players expect the authority to require removal, $\mathrm{G}$ always acceding to the request, and there being no appeals in equilibrium. ${ }^{2}$

The equilibrium characterizations in the two extreme cases of $\beta=0$ and $\beta=1$ do not capture the real situations where individuals' removal requests sometimes get rejected by the search engine. Even if we assume $\beta \in(0,1)$ in the complete information game, there is no equilibrium that can represent the real cases where individuals do not appeal to local data protection authorities when their requests are rejected by the search engine. These considerations provide justification for our assumption of the asymmetric information game.

\section{B2. The Impact of the RTBF Expansion}

We now discuss the impact of changing the RTBF scope in the game under complete information. As in the main text, we assume that expansion of the RTBF is represented by a combination of an upward shift in $q$ to one and an increase in $l$ (Assumption 1). Under this assumption, we describe the effect of the RTBF expansion on the probability of link removal for each possible case in terms of $\beta$. Note that if $q$ increases, then $\frac{C_{P}}{q h}$ and $\frac{c+C_{P}}{q h}$ decrease; and if $l$ increases, then $\frac{l-C_{G}}{l}$ increases, given the parameter values of $c, h, C_{P}$, and $C_{G}$.

First suppose that $\frac{c+C_{P}}{q h} \leq \frac{l-C_{G}}{l}$. Then the parameter range of $\beta$ given other parameter values for which the equilibrium in case (2-ii) arises, i.e., $\left[\frac{c+C_{P}}{q h}, \frac{l-C_{G}}{l}\right]$, expands to $\left[\frac{c+C_{P}}{h}, \frac{l^{\prime}-C_{G}}{l^{\prime}}\right]$ where $l^{\prime}>l$. For any case of $\beta \in\left[\frac{c+C_{P}}{h}, \frac{c+C_{P}}{q h}\right)$, the probability of link removal is zero before the expansion (because there is no claim in equilibrium); after the expansion, the probability of link removal becomes $\beta>0$. For any case of $\beta \in\left(\frac{l-C_{G}}{l}, \frac{l^{\prime}-C_{G}}{l^{\prime}}\right]$, the probability of link removal is one before the expansion (because $\mathrm{G}$ always accepts in equilibrium upon claim); after the expansion, it becomes $\beta<1$. For any "interior" case

\footnotetext{
${ }^{2}$ We thank an anonymous editor for alerting us to this case.
} 
of $\beta \in\left[\frac{c+C_{P}}{q h}, \frac{l-C_{G}}{l}\right]$, the probability of link removal, which equals $\beta$, remains unchanged after the expansion. For any case of $\beta \in\left(0, \frac{c+C_{P}}{h}\right)$ and for any case of $\beta \in\left(\frac{l^{\prime}-C_{G}}{l^{\prime}}, 1\right)$, the probabilities of link removal are zero and one, respectively, which also remain unchanged after the expansion.

Now suppose that $\frac{l-C_{G}}{l}<\frac{c+C_{P}}{q h}$. Then case (2-ii) never arises before the expansion. The comparison becomes more cumbersome depending on parameter values, but the logic is the same as in the previous consideration. Roughly put, of the cases with $\beta$ close to the value of threshold that distinguishes case (2-i) and case (3), the probability of link removal increases from zero to $\beta$ for a case of a relatively low $\beta$ while it decreases from one to $\beta$ for a case of a higher $\beta$. For all other cases, the probability of link removal remains to be zero, $\beta$, or one.

In sum, the effect of global expansion on the probability of link removal for each potential removal claim (in both the intensive and extensive margins) depends crucially on the parameter values associated with the potential claim. What is essential is that global expansion can lessen the possibility of each removal claim resulting in the removal of the links in a symmetric information setting, as in our asymmetric information game. Accordingly, the overall incidence of link removals can either increase or decrease, conforming to our key result in the main text.

We conclude this appendix by noting that the likelihood of $\mathrm{P}$ prevailing on appeal $\beta$ is taken as given. As we briefly discuss in Section 6 of the main text, the actual decision of a data protection agency or a court would depend on the payoffs to the petitioner and the search engine as well as on the payoffs to the search engine's users. The parameter $\beta$ represents the expected ruling of the data protection agency, but our model does not impose any restrictions on how $\beta$ might depend on the relevant parties' payoffs. If we take $\beta$ as a function of $q, h, l$, and $S$, then the impact of global expansion should also reflect a change in $\beta$. This consideration can be easily accommodated in our analysis under complete information; however, it only complicates the analysis without adding commensurate insight. 


\section{Appendix C}

\section{Illustrations and Discussions}

\section{C1. Illustration of Corollary 1}

Panel (a) of Figure $\mathrm{C} 1$ depicts two pairs of $(q, l)$ with different initial levels of $q$. The probability of link removal decreases for the pair labeled (1) but increases for the pair labeled (2), by a shift in $q$ to one and arbitrary increases in $l$. Panel (b) of Figure C1 shows that the probability of link removal can either increase or decrease depending on the size of an increase in $l$ for the same initial $q$.

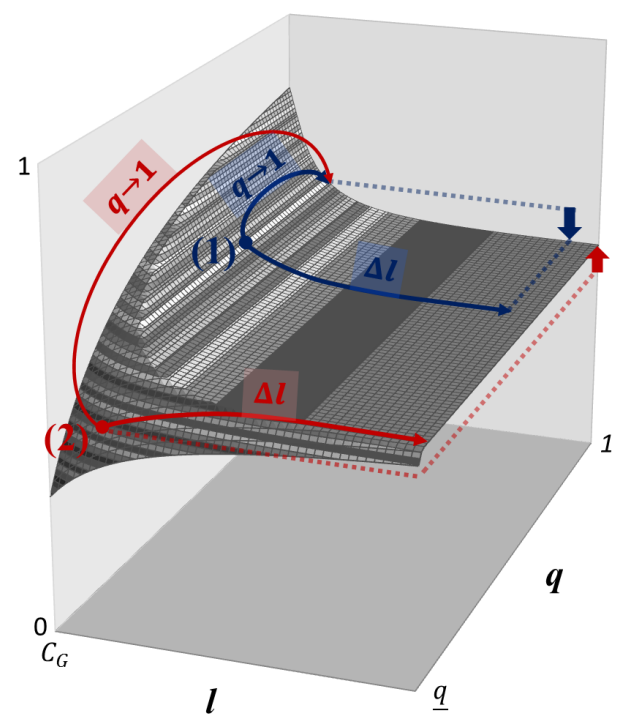

(a) Different $q$ 's with arbitrary increases in $l$

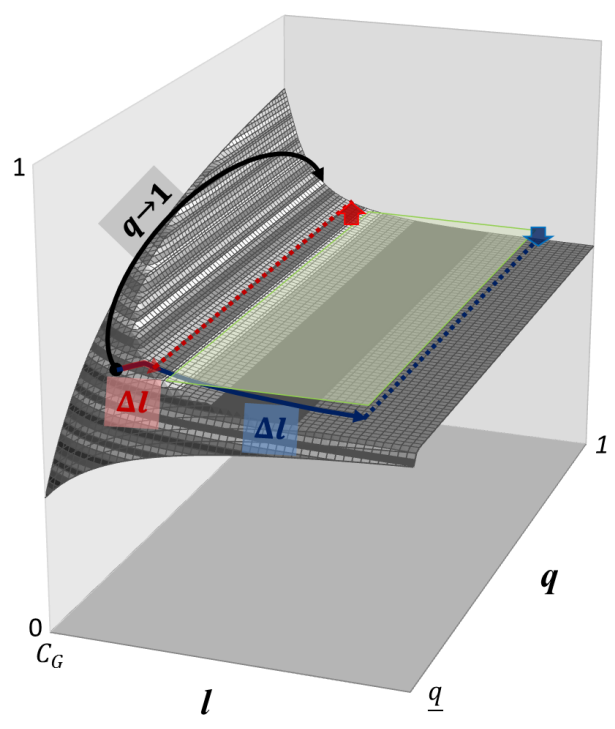

(b) Same $q$ 's with different increases in $l$

Figure C1. The effect of the global expansion on the probability of link removal

\section{C2. Measuring the Overall Incidence of Link Removals across All Potential Claims}

To fully predict how the global expansion affects the overall incidence of link removals across all potential removal claims, it is necessary to carry out the following tasks. For each potential link removal claim, the payoff-relevant parameter values may vary. So for each removal claim represented by the parameter $h$, we estimate a triplet $\left(q, l, l^{\prime}\right)$ where $l^{\prime}$ is the amount of search engine's expected loss after the expansion. For simplicity, we 
assume that $c, C_{P}$, and $C_{G}$ are fixed constants across all players. Then we assume an appropriate joint distribution of $\left(q, l, l^{\prime}\right)$ for a given value of $h$ as well as a distribution of $h$. For example, let $\Psi\left(q, l, l^{\prime} \mid h\right)$ denote the conditional cumulative distribution function of $\left(q, l, l^{\prime}\right)$ given $h$, and $\Phi(h)$ denote the cumulative distribution function for $h$ in the cross section of petitioners. If we denote $\Delta\left(q, l, l^{\prime} \mid h\right) \equiv \operatorname{Pr}(q, l \mid h)-\operatorname{Pr}\left(1, l^{\prime} \mid h\right)$ as the change in the probability of link removal by the global expansion for a given case of removal request, then the $\int_{h}\left[\int_{\left(q, l, l^{\prime}\right)} \Delta\left(q, l, l^{\prime} \mid h\right) d \Psi\left(q, l, l^{\prime} \mid h\right)\right] d \Phi(h)$ measures the overall change in the probability of link removal across all possible RTBF cases.

Gauging this measure complicates the exposition without adding any new insights. To further evaluate how global expansion changes the total number of the links that are removed among all of the removal claims, we need to estimate the change in the number of claims in the extensive margin as well as the number of the links that are requested for removal (which may vary across claims); this falls beyond the scope of our methodology. We note that the comparative statics results on the equilibrium probability of link removal for each given case in the main text are sufficient to provide some conceivable effects of the RTBF expansion on link removal incidence.

\section{C3. A Schematic Representation of the Main Argument}

Figure C2 shows a schematic representation of our main argument in the paper.

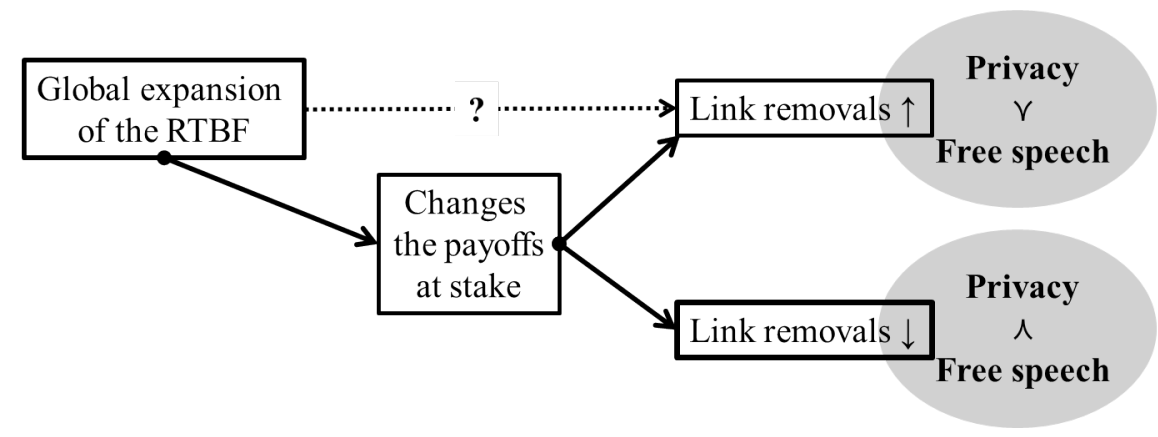

Figure C2. A schematic representation of the equilibrium properties of the global expansion 


\section{Appendix D}

\section{The RTBF Game with Broad Interpretations of Appeal Costs}

\section{D1. Effects of Appeal Costs on the Equilibrium Probability of Link Removal}

Given the broader interpretations of the players' appeal costs in Section 4.3, the magnitude of such costs are another important factors that shape the search engine's decision to reject, the petitioner's decision to appeal, and correspondingly the probability of link removal. As a complementary analysis to Section 4 of the main text, this appendix examines the effect of changes in appeal costs on the probability of link removal in the unique equilibrium where P claims.

We let $\bar{C}_{P} \equiv \mathbb{E}[\beta] q h$ denote the upper bound on $C_{P}$ implied by Condition 1 ; let $\bar{C}_{G} \equiv l$ denote the upper bound on $C_{G}$ implied by Condition 2 ; and let $C_{P}^{*}$ and $C_{G}^{*}$ denote the values of $C_{P}$ and $C_{G}$, respectively, such that $\beta_{G}^{*}=\beta^{*}$, each defined given other parameter values. The following result offers the comparative statics with regard to $C_{G}$ and $C_{P}$.

Proposition D1. (a) The equilibrium probability of link removal is increasing in $C_{G}$ if $C_{G} \in\left(0, C_{G}^{*}\right)$ but is decreasing in $C_{G}$ if $C_{G} \in\left[C_{G}^{*}, \bar{C}_{G}\right)$. (b) The equilibrium probability of link removal is not affected by $C_{P}$ if $C_{P} \in\left(0, C_{P}^{*}\right)$ but is decreasing in $C_{P}$ if $C_{P} \in\left[C_{P}^{*}, \bar{C}_{P}\right)$.

Proof. We first begin with how the best responses of G vary with appeal costs:

$$
\frac{d \beta_{G}^{*}}{d C_{G}}<0, \quad \frac{d \beta^{*}}{d C_{G}}=0 ; \quad \frac{d \beta_{G}^{*}}{d C_{P}}=0, \quad \frac{d \beta^{*}}{d C_{P}}>0
$$

Because $\beta_{G}^{*}=\frac{l-C_{G}}{l}$, an increase in $C_{P}$ has no effect on $\beta_{G}^{*}$, whereas an increase in $C_{G}$ decreases $\beta_{G}^{*}$. On the other hand, an increase in $C_{G}$ has no effect on $\beta^{*}$ defined by $\mathbb{E}[\beta \beta \leq$ $\left.\beta^{*}\right] q h=C_{P}$ but an increase in $C_{P}$ increases $\beta^{*}$.

Part (a): The case of $C_{G} \in\left(0, C_{G}^{*}\right)$ corresponds to when $\beta_{G}^{*}>\beta^{*}$ where G's optimal cutoff type is $\beta_{G}=\beta_{G}^{*}$ and $p_{a}=1$. Differentiation of the equilibrium probability of link removal with respect to $C_{G}$ yields $\left[-\left(1-\beta_{G}^{*}\right) f\left(\beta_{G}^{*}\right)\right] \frac{d \beta_{G}^{*}}{d C_{G}}>0$, because the term in the bracket is 
strictly negative $\left(\beta_{G}^{*}<1\right.$ for $\left.C_{G}>0\right)$ and $\frac{d \beta_{G}^{*}}{d C_{G}}<0$ by (D1). The case of $C_{G} \in\left[C_{G}^{*}, \bar{C}_{G}\right)$ corresponds to when $\beta_{G}^{*} \leq \beta^{*}$ where $\beta_{G}=\beta^{*}$ and $p_{a}=\frac{l}{\beta^{*} l+C_{G}}$. Because $\frac{d \beta^{*}}{d C_{G}}=0$ by (D1), all the terms in the equilibrium probability of link removal, $\left(1-F\left(\beta^{*}\right)\right)+F\left(\beta^{*}\right) p_{a} \mathbb{E}\left[\beta \mid \beta \leq \beta^{*}\right.$, remain unchanged by an increase in $C_{G}$ except for $p_{a}=\frac{l}{\beta^{*} l+C_{G}}$. Because this $p_{a}$ is decreasing in $C_{G}$, the equilibrium probability of link removal falls.

Part (b): The case of $C_{P} \in\left(0, C_{P}^{*}\right)$ corresponds to when $\beta_{G}^{*}>\beta^{*}$. Both $\beta_{G}=\beta_{G}^{*}$ and $p_{a}=1$ are not affected by $C_{P}$. Hence, the equilibrium probability of link removal in this case, $\left(1-F\left(\beta_{G}^{*}\right)\right)+F\left(\beta_{G}^{*}\right) \mathbb{E}\left[\beta \mid \beta \leq \beta_{G}^{*}\right]$, remains constant by any small change in $C_{P}$. For $C_{P} \in\left[C_{P}^{*}, \bar{C}_{P}\right)$ that corresponds to when $\beta_{G}^{*} \leq \beta^{*}$, we have:

$$
\frac{d P r(\text { "link-removal") }}{d C_{P}}=\left[-\left(1-p_{a} \beta^{*}\right) f\left(\beta^{*}\right)-F\left(\beta^{*}\right)\left(p_{a}\right)^{2}\left(\beta^{*}-\frac{1}{F\left(\beta^{*}\right)} \int_{0}^{\beta^{*}} F(x) d x\right)\right] \frac{d \beta^{*}}{d C_{P}} .
$$

The bracketed term is strictly negative while $\frac{d \beta^{*}}{d C_{P}}>0$. Hence, the equilibrium probability of link removal decreases with a small increase in $C_{P}$ in this case. Q.E.D.

An interesting observation is that the effect of an increase in $C_{G}$ (resp. $\left.C_{P}\right)$ is substantively equivalent to the effect of a decrease in $l$ (resp. q). We briefly point out the key forces at work underlying Proposition D1. For a higher G's appeal cost, an increased probability of G's acceptance (higher $1-F\left(\beta_{G}^{*}\right)$ ) primarily contributes to a greater chance of link removal when $C_{G}$ is small enough; whereas when $C_{G}$ is sufficiently high, the petitioner's lower probability of appealing solely leads to less chance of link removal. For a higher P's appeal cost up to a certain level, the probability of $\mathrm{G}^{\prime}$ 's rejection $\left(F\left(\beta_{G}^{*}\right)\right)$ is already high enough so that $\mathrm{P}$ believes he still has a fair chance of winning on appeal to compensate for his higher appeal cost, maintaining his commitment to appeal $\left(p_{a}=1\right)$; hence leaving the equilibrium probability of link removal unchanged. However when $C_{P}$ is sufficiently high, $\mathrm{P}$ proceeds to an appeal less often with a higher appeal cost, inducing less probability of G's acceptance, which essentially leads to a decrease in the equilibrium probability of link removal.

Figure D1 illustrates the combined effects of changes in $C_{P}$ and $C_{G}$ on the equilibrium 
probability of link removal. ${ }^{3}$ If we extend Assumption 1 to also allowing for increases in $C_{G}$ and $C_{P}$ to represent the global expansion, our key implication that the expansion can decrease the incidence of link removals remains qualitatively intact.

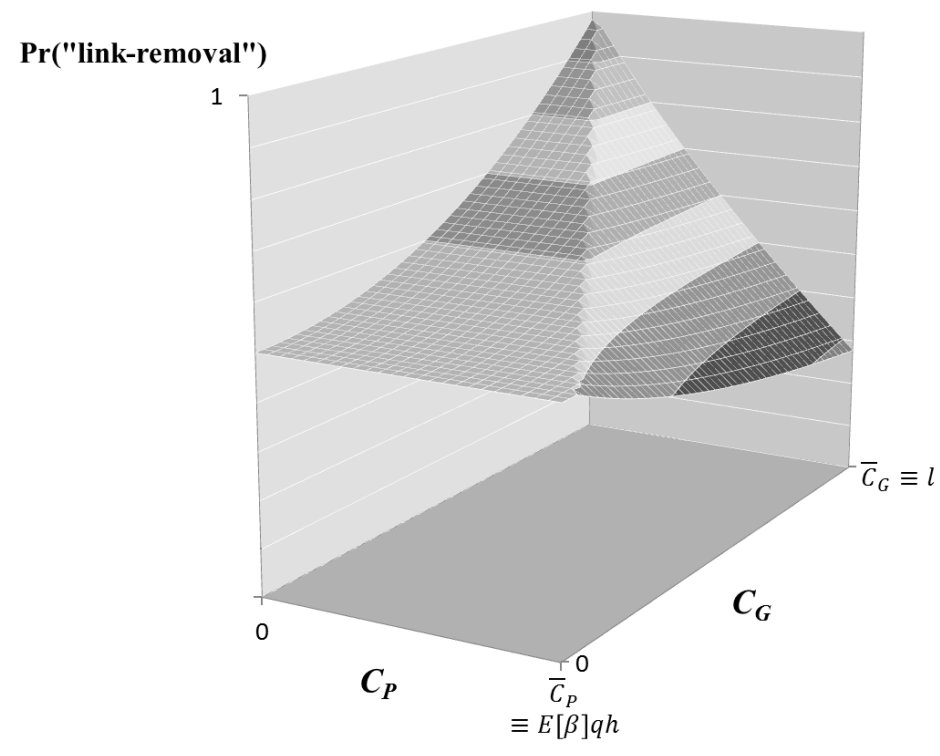

Figure D1. The effects of $C_{P}$ and $C_{G}$ on the equilibrium probability of link removal in the claim equilibrium under Conditions 1 and 2

\section{D2. Effects of Legal Rules on the Equilibrium Probability of Link Removal}

One may consider models with alternative legal rules governing the allocation of appeal (litigation) costs. This consideration is especially useful if we interpret the petitioner's appeal as bringing the case to a lawsuit. In our setup, we assume the American rule that each party bears his or her own litigation costs in case of a trial regardless of the trial's outcome. But in some cases, a losing party might bear all of the litigation costs - the legal arrangement that is referred to as the British rule. The framework we have developed is amenable to examining our game under the British rule by modifying accordingly the parties' expected payoffs from litigation, which are summarized in Table D1.

The equilibrium of the RTBF game under the British rule can be characterized similarly

\footnotetext{
${ }^{3}$ The figure is obtained for an example with a uniform $F(\cdot), q=1, h=50$, and $l=50$. The pattern of the figure is generic for a claim equilibrium given any other parameters that satisfy Conditions 1 and 2 .
} 
Table D1. The expected payoffs from litigation under different rules on litigation costs

\begin{tabular}{c|c|c} 
& Under American rule & Under British rule \\
\hline \hline $\mathrm{P}$ & $-(1-\beta q) h-c-C_{P}$ & $-(1-\beta q) h-(1-\beta)\left(C_{P}+C_{G}\right)-c$ \\
$\mathrm{G}$ & $-\beta l-C_{G}$ & $-\beta\left(l+C_{G}+C_{P}\right)$
\end{tabular}

as in Propositions 1 and 2. We characterize equilibrium strategies in the subgame after P's claim as follows, where the proof is analogous to the proof of Proposition 1.

Corollary 2. Under the condition that $\mathbb{E}[\beta]\left(q h+C_{P}+C_{G}\right)>C_{P}+C_{G}$ :

(i) If $\beta_{G}^{*}>\beta^{*}$, then Gs of type $\beta>\beta_{G}^{*}$ accept the claim; Gs of type $\beta \leq \beta_{G}^{*}$ reject it, and $\mathrm{P}$ appeals with probability one;

(ii) If $\beta_{G}^{*} \leq \beta^{*}$, then Gs of type $\beta>\beta^{*}$ accept the claim; Gs of type $\beta \leq \beta^{*}$ reject it, and $\mathrm{P}$ appeals with probability $p_{a}=\frac{l}{\beta^{*}\left(l+C_{G}+C_{P}\right)} \in(0,1]$, where $\beta_{G}^{*} \equiv \frac{l}{l+C_{G}+C_{P}}$ and $\beta^{*}$ solves $\mathbb{E}\left[\beta \mid \beta \leq \beta^{*}\right]\left(q h+C_{P}+C_{G}\right)=C_{P}+C_{G}$.

All of the key insights of our comparative statics in Section 4 continue to hold under the British rule. Thus the general conclusion of the main text with regard to the impact of the global expansion are robust to alternative specifications of legal rules on litigation costs.

However, how equilibrium behavior is affected by a change from the American rule to the British rule is erratic. The reason for this ambiguity is because whether G's optimal cutoff type under the British rule is higher or lower than that under the American rule crucially depends on the primitives of the model, and the change is not monotonic. Therefore, a change from the American rule to the British rule might increase, decrease, or have no effect on the equilibrium probability of link removal, depending on the parameters of the model.

As an illustrative example, Figure D2 shows how the probability of link removal is affected by legal rules on litigation costs in terms of $C_{P} \cdot{ }^{4}$ The thresholds and the upper bounds are defined similarly as before with the superscripts $A$ and $B$ that indicate the American rule and the British rule respectively.

\footnotetext{
${ }^{4}$ The figure is obtained for an example with a uniform $F(\cdot), q=1, h=50, l=50$, and $C_{G}=10$.
} 


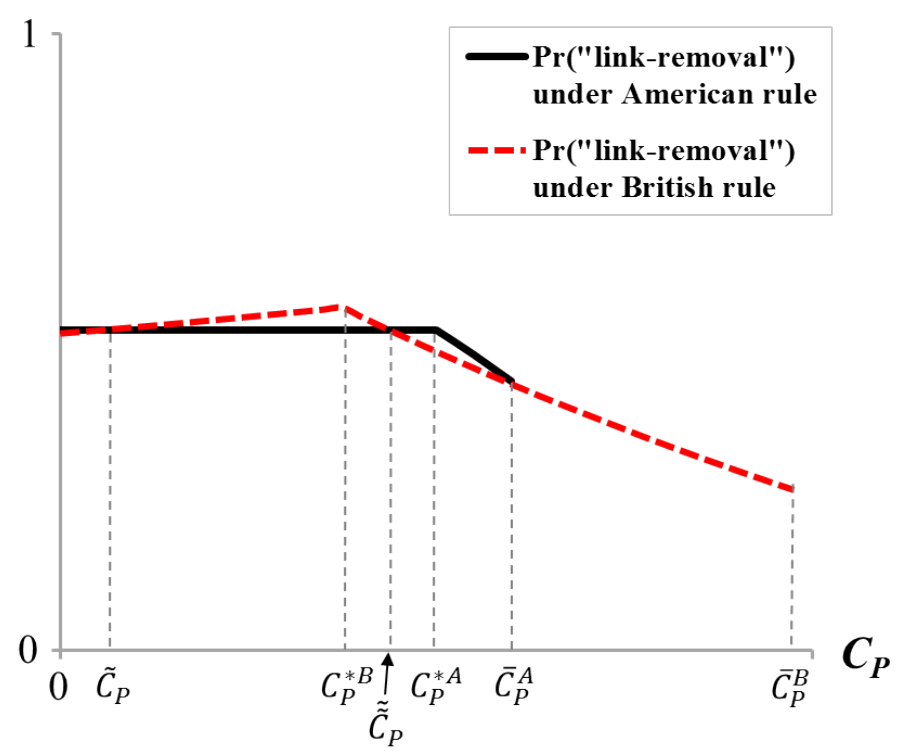

Figure D2. The effects of legal rules on the equilibrium probability of link removal

We can see from the figure that the probability of link removal under the British rule is lower when $C_{P} \in\left(0, \tilde{C}_{P}\right)$ or when $C_{P} \in\left(\tilde{\tilde{C}}_{P}, \bar{C}_{P}^{A}\right)$, but is higher when $C_{P} \in\left(\tilde{C}_{P}, \tilde{\tilde{C}}_{P}\right)$, compared to that under the American rule. When $C_{P} \geq \bar{C}_{P}^{A}, \mathrm{P}$ never claims in the unique sequential equilibrium of the RTBF game under the American rule. So the probability of link removal is trivially zero in this case, whereas the probability of link removal in the claim equilibrium of the game under the British rule is positive up to $C_{P}=\bar{C}_{P}^{B}$ where $\bar{C}_{P}^{B}>\bar{C}_{P}^{A}$. 